\title{
High Angular Momentum Halo Gas: A Feedback and Code-independent Prediction of LCDM
}

\author{
Kyle R. Stewart ${ }^{1}$, Ariyeh H. Maller ${ }^{2,3}$, Jose Oñorbe ${ }^{4}$, James S. Bullock ${ }^{5,6}$, M. Ryan Joung ${ }^{7}$, Julien Devriendt ${ }^{8}$, Daniel Ceverino ${ }^{9}$, \\ Dušan Kereš ${ }^{10}$, Philip F. Hopkins ${ }^{11}$, and Claude-André Faucher-Giguère ${ }^{12}$ \\ ${ }^{1}$ Department of Mathematical Sciences, California Baptist University, 8432 Magnolia Ave., Riverside, CA 92504, USA \\ ${ }^{2}$ Department of Physics, New York City College of Technology, 300 Jay St., Brooklyn, NY 11201, USA \\ ${ }^{3}$ Department of Astrophysics, American Museum of Natural History, Central Park West at 79th St., New York, NY 10024, USA \\ ${ }^{4}$ Max-Planck-Institut für Astronomie, Königstuhl 17, D-69117 Heidelberg, Germany \\ ${ }^{5}$ Center for Cosmology, Department of Physics and Astronomy, The University of California at Irvine, Irvine, CA 92697, USA \\ ${ }^{6}$ Center for Galaxy Evolution, Department of Physics and Astronomy, The University of California at Irvine, Irvine, CA 92697, USA \\ 7 Department of Astronomy, Columbia University, New York, NY 10027, USA \\ ${ }_{9}^{8}$ Department of Physics, University of Oxford, The Denys Wilkinson Building, Keble Rd., Oxford OX1 3RH, UK \\ ${ }^{9}$ Zentrum für Astronomie der Universität Heidelberg, Institut für Theoretische Astrophysik, Albert-Ueberle-Str. 2, \\ D-69120 Heidelberg, Germany \\ ${ }^{10}$ Department of Physics, Center for Astrophysics and Space Sciences, University of California at San Diego, 9500 Gilman Dr., \\ La Jolla, CA 92093, USA \\ ${ }^{11}$ California Institute of Technology, 1200 E. California Blvd., Pasadena, CA 91125, USA \\ ${ }^{12}$ Department of Physics and Astronomy and CIERA, Northwestern University, 2145 Sheridan Rd., Evanston, IL 60208, USA \\ Received 2016 June 27; revised 2017 April 3; accepted 2017 April 17; published 2017 June 30
}

\begin{abstract}
We investigate angular momentum acquisition in Milky Way-sized galaxies by comparing five high resolution zoom-in simulations, each implementing identical cosmological initial conditions but utilizing different hydrodynamic codes: Enzo, Art, Ramses, Arepo, and Gizmo-PSPH. Each code implements a distinct set of feedback and star formation prescriptions. We find that while many galaxy and halo properties vary between the different codes (and feedback prescriptions), there is qualitative agreement on the process of angular momentum acquisition in the galaxy's halo. In all simulations, cold filamentary gas accretion to the halo results in $\sim 4$ times more specific angular momentum in cold halo gas $\left(\lambda_{\text {cold }} \gtrsim 0.1\right)$ than in the dark matter halo. At $z>1$, this inflow takes the form of inspiraling cold streams that are co-directional in the halo of the galaxy and are fueled, aligned, and kinematically connected to filamentary gas infall along the cosmic web. Due to the qualitative agreement among disparate simulations, we conclude that the buildup of high angular momentum halo gas and the presence of these inspiraling cold streams are robust predictions of Lambda Cold Dark Matter galaxy formation, though the detailed morphology of these streams is significantly less certain. A growing body of observational evidence suggests that this process is borne out in the real universe.
\end{abstract}

Key words: galaxies: evolution - galaxies: formation - galaxies: halos - hydrodynamics - methods: numerical

\section{Introduction}

In the standard Lambda Cold Dark Matter (LCDM) picture of galaxy formation, gas accreting onto a growing dark matter halo shock-heats to the virial temperature of the halo, giving the gas time to virialize and eventually cool out of the hot gaseous halo and sink into the central galaxy (Rees \& Ostriker 1977; Silk 1977; White \& Rees 1978; White \& Frenk 1991; Maller \& Bullock 2004). Under this picture of galaxy growth, it is expected that the resulting angular momentum distribution of galaxies should mimic the spin of their dark matter, resulting in rotationally supported galaxy disks (and presumably hot gaseous halos as well) that are proportional to the spin of the dark matter halo (Fall \& Efstathiou 1980; Mo et al. 1998), which has been well-studied in dissipationless $N$-body simulations and semi-analytic merger trees (e.g., Bullock et al. 2001; Maller et al. 2002; Vitvitska et al. 2002; Avila-Reese et al. 2005; D'Onghia \& Navarro 2007; Bett et al. 2010; Muñoz-Cuartas et al. 2011; Ishiyama et al. 2013; Trowland et al. 2013; Kim et al. 2015).

However, recent advances in hydrodynamic simulations and galaxy formation theory have increasingly emphasized the importance of "cold flows"- gas accretion onto galaxy halos via filamentary streams with cooling times shorter than the compression time for establishing a stable shock ${ }^{13}$, either when the halo is below a critical mass threshold, or even for massive halos at sufficiently high redshift (e.g., Binney 1977; Kereš et al. 2005; Dekel \& Birnboim 2006; Ocvirk et al. 2008; Brooks et al. 2009; Dekel et al. 2009; Faucher-Giguère \& Kereš 2011; Faucher-Giguère et al. 2011; Stewart et al. 2011a; van de Voort et al. 2011, 2015; Hobbs et al. 2015). In the cold flow paradigm, gas that is accreted in the cold mode tends to have specific angular momentum considerably higher than the dark matter (Chen et al. 2003; Sharma \& Steinmetz 2005; Agertz et al. 2009; Kereš et al. 2009; Kereš \& Hernquist 2009; Brook et al. 2011; Kimm et al. 2011; Stewart et al. 2011b), inconsistent with the previous picture of galaxy angular momentum buildup. The resulting angular momentum of the stellar disk may be rather different from that of the accreted gas because of feedback effects (Maller \& Dekel 2002; Brook et al. 2011).

\footnotetext{
13 Some recent moving-mesh simulations have called into question whether these cold streams deliver unshocked gas to the galaxy without heating in the inner regions of the halo (e.g., Torrey et al. 2012; Nelson et al. 2013, 2015, 2016). As our focus in this work is on gas accretion into the halo, not the eventual transition from the halo to the galaxy, this distinction should have minimal impact on the topics discussed here.
} 
As a result of this changing paradigm for cosmological gas accretion and galaxy growth (for a recent review, see Stewart 2016), a new scenario of angular momentum acquisition in galaxies and galaxy halos seems to be emerging. In this picture (Kimm et al. 2011; Pichon et al. 2011; Stewart et al. 2011b, 2013; Codis et al. 2012, 2015; Danovich et al. 2012, 2015; Prieto et al. 2015; Tillson et al. 2015), the particularly high angular momentum of cold flow gas is related to its coherent, filamentary origin, coupled with the specific geometry of the cosmic web in the environment of a given galaxy. These filamentary cold flows deliver significant angular momentum to galaxy halos, with the cold gas orbiting for $\sim 1-2$ dynamical times before spiraling into the central galaxy. At any given time, galaxy disks typically have lower spin than halo gas, owing to the fact that the specific angular momentum of infalling material increases with time. Halo gas is "younger" and this correlates with higher spin.

Importantly, this scenario is predictive. The high-spin halo gas is often (but not always) coherent in its spin direction, with inspiraling cold streams often forming a thick planar structure of high angular momentum cool gas that co-rotates with the central disk. It is important to emphasize that while this extended gas tends to rotate, it is not angular-momentum supported. Rather, this gas usually spirals in on $\sim 2$ dynamical times. Though not perfectly aligned with the orientation of the galactic disk, the inspiraling halo gas usually has coherent rotation along a preferred plane.

Encouragingly, an increasing number of observations have begun to demonstrate the abundance of high angular momentum material in galaxy halos, qualitatively consistent with this emerging theoretical picture. In the local universe, some of these observations include detection of high angular momentum extended $\mathrm{H}$ I disks and XUV disks (Oosterloo et al. 2007; Christlein \& Zaritsky 2008; Sancisi et al. 2008; Lemonias et al. 2011; Holwerda et al. 2012), as well as low metallicity high angular momentum gas (presumably from fresh accretion) in polar ring galaxies (Spavone et al. 2010). There is even indication that local extended H I disks may be environmentally dependent on the galaxy's filamentary environment (Courtois et al. 2015). At moderate redshift $(z \sim 0.5-1.5)$, numerous absorption line studies of the circumgalactic medium of galaxies have begun to emphasize the bimodal properties of absorbers, where absorption along the galaxy's major axis tends to show high angular momentum (co-rotating) inflow, and absorption along the galaxy's minor axis shows observational signatures of outflow (Kacprzak et al. 2010, 2012a, 2012b; Bouché et al. 2012, 2013; Crighton et al. 2013; Nielsen et al. 2015; Bouché et al. 2016; Diamond-Stanic et al. 2016; Ho et al. 2017). At higher redshift $(z \sim 2-3)$, kinematic studies of Ly $\alpha$ "blobs" have observed large-scale rotation consistent with high angular momentum cold gas accretion (Martin et al. 2014; Prescott et al. 2015). There are also recent detections of massive protogalactic gaseous disks kinematically linked to gas inflow along cosmic filaments, strikingly similar to the theoretical "cold flow disk" structure (Martin et al. 2015, 2016).

In this context, it is important that we ascertain how robust the predictions of these cosmological simulations are-a difficult task, considering that many properties of simulated galaxies depend sensitively on the implementation of uncertain subgrid physics models such as gas cooling, star formation, radiation pressure, and supernova feedback (e.g., Thacker \&
Couchman 2000; Kay et al. 2002; Gnedin et al. 2011; Piontek \& Steinmetz 2011; Martizzi et al. 2012; Scannapieco et al. 2012; Agertz et al. 2013; Vogelsberger et al. 2013, 2014; Ceverino et al. 2014; Genel et al. 2015; Marasco et al. 2015; Agertz \& Kravtsov 2016). In addition, even with identical subgrid implementations, there are inherent numerical advantages and disadvantages between different hydrodynamic code implementations - for example, Lagrangian smoothed particle hydrodynamic $(\mathrm{SPH})$ versus Eulerian grid codes-that result in artificial differences between galaxies simulated with different codes (e.g., Morris 1996; Agertz et al. 2007; Wadsley et al. 2008; Cullen \& Dehnen 2010; Hahn et al. 2010; Springel 2010; Hopkins 2015; Richardson et al. 2016).

In order to test the validity of the emerging cold flow picture of angular momentum acquisition, we must ascertain the dependency of these predictions on the use of different numerical techniques and a variety of cutting-edge subgrid physics models. In this paper, we run five hydrodynamic zoomin simulations of a Milky Way-sized galaxy, each with identical cosmological initial conditions but with different codes: Enzo, Ramses, Art, Arepo, and Gizmo-PSPH, each implemented with recent subgrid physics models. In order to ensure uniform analysis for different hydrodynamic codes, we utilize the analysis software yt (which allows a single analysis routine to be run on different code architectures; Turk et al. 2011) to explore the angular momentum content of halo gas and whether or not the expected "cold flow disk" prediction is robust across these disparate platforms. We introduce the simulations in Section 2, present our main results from the comparison Sections 3-5, finding that the same qualitative picture of high angular momentum halo gas in the form of codirectional inspiraling cold streams (which do occasionally take the form of cold flow disks) is present in all simulations-a seemingly natural consequence of filamentary gas accretion in LCDM. We discuss the implications of these results and the growing observational evidence of their existence in Section 6 and summarize and conclude in Section 7.

\section{The Simulations}

\subsection{Overview}

The simulations used in this paper are all part of the Scylla Multi-Code Comparison Project. This project resimulates a Milky Way halo mass zoom-in simulation (originally performed by Ryan Joung with Enzo in Joung et al. 2012) using other cosmological hydrodynamic codes. While we focus on the redshift range $1<z<3$ in this work, we note that the resulting disk-type galaxy and its gaseous halo have already been studied in detail at low redshift (e.g., Fernández et al. 2012; Joung et al. 2012). Of particular importance to this work, Fernández et al. (2012) determined that the mass (in HI), covering fraction, and spatial distribution of the cold gas halo at $z=0$ are consistent with existing observations of nearby spiral galaxies.

The codes are all run with their recent ${ }^{14}$ subgrid models in order to compare state of the art simulations across codes. Thus, the project is much like the Aquilla code comparison (Scannapieco et al. 2012) but with higher resolution. Our resolution is similar to the Agora code comparison project

\footnotetext{
${ }^{14}$ Inevitably, however, there are bound to be further improvements to some of the subgrid models during the time it took to run the simulations, and analyze and publish the results.
} 
(Kim et al. 2014), but that project is seeking to use uniform physics while we are running each code as it has been used for other science papers. The codes used here are Enzo, Art, Ramses, Arepo, and Gizmo-PSPH. For all runs, the cosmology, dark matter particle mass, and box size are identical-the box is $25 \mathrm{Mpc} h^{-1}$ across, with a much smaller region simulated at high resolution, using dark matter particles of mass $1.75 \times 10^{5} M_{\odot}$. All adaptive mesh refinement (AMR) codes reach the same maximum refinement of $95 \mathrm{~h}^{-1}$ comoving pc, which is identical to the force resolution of the Lagrangian codes, with the exception of gas particles in Gizmo-PSPH, which uses an adaptive gravitational softening with a minimum value of $14 h^{-1}$ comoving pc. A flat cosmology consistent with WMAP5 (Komatsu et al. 2009) is used throughout, with $\Omega_{m}=0.279, \Omega_{\Lambda}=0.721, \Omega_{b}=0.046$, $h=0.70, \sigma_{8}=0.82$, and $n_{s}=0.96$.

The initial conditions for the original Enzo run (Joung et al. 2012) were generated with the code Grafic ${ }^{15}$ (Bertschinger 2011) with a starting redshift of $z=99$. The same code with the same seed was used to generate the initial conditions for the Ramses run. For all other runs, the dark matter particles from the Enzo run were used to determine the initial conditions. That is, the dark matter particles were set identical to those in the Enzo run and baryons were added based on the dark matter distribution (no separate transfer function). We expect these differences to be negligible by the redshift where galaxies are forming.

Based on the cosmological model specified above, all Lagrangian codes set the gas mass resolution $\left(m_{\mathrm{gas}}=\right.$ $\left.3.3 \times 10^{4} M_{\odot}\right)$ relative to the dark matter particle mass $\left(m_{\mathrm{DM}}=1.75 \times 10^{5} M_{\odot}\right)$. Table 1 outlines many of the pertinent details for each code, including star formation (SF) density thresholds and efficiency parameters, epoch of reionization, UV background model, and the type of stellar feedback model adopted. Below, we describe the gas cooling and feedback physics of each individual run in more detail and include references to recently published science papers that utilize similar subgrid physics models as those implemented here.

For all analyses that follow, we make the distinction between "cold" and "hot" gas by a temperature cutoff of 250,000 K (commonly used as the distinction between "cold-mode" and "hot-mode" gas accretion; e.g., Kereš et al. 2005, 2009; Stewart et al. 2011b, 2013). Using the Rockstar halo finder (Behroozi et al. 2013b), we calculate the virial radius of each simulation at each output redshift, finding that the halo-finding algorithms produce slightly different virial radii at the same redshift for different simulations ( $\pm 5 \%$ from the mean). For the sake of identical comparison between codes, we therefore utilize a fitting function for $R_{\mathrm{vir}}(z)$ that averages over all simulations to adopt an identical estimated virial radius for all simulations (at a given redshift), which varies from 68 physical $\mathrm{kpc}$ at $z=3$ to 171 physical $\mathrm{kpc}$ at $z=1$.

In order to guarantee uniform analysis for the varied code architectures and file formats, all analyses presented here have been performed utilizing the $\mathrm{yt}^{16}$ analysis software (Turk et al. 2011; Turk \& Smith 2011; Turk 2013), an open source project that has been developed and is continually being maintained and improved by the astrophysical community for the intended purpose of supporting cross-code compatible hydrodynamic

\footnotetext{
${ }^{15}$ http://web.mit.edu/edbert/

16 http://yt-project.org
}

analysis routines. In plots showing images of the gas distribution, we use a slightly older version of yt (version yt3.2.3) because after that version yt updated the way gas particles are deposited into cells. Prior to the update, yt used clouds-in-cells deposition to determine the gas properties of a cell, while after the update the sph smoothing kernel is used instead. Although this update gives more accurate deposition for sph particles, it is very inaccurate for Arepo where the particle size relates to the volume of the cells from the Voronoi tessellation and not a smoothing length. The clouds-in-cells deposition gives adequate results for both methods so we use it for images. For quantitative analysis, we use the gas particles for both Arepo and Gizmo-PSPH so that no deposition into cells is required.

\subsection{Enzo}

The Enzo (Bryan et al. 2014) run serves as the basis for the Scylla simulation suite, and was performed in 2010 by Ryan Joung and discussed in Joung et al. (2012), Fernández et al. (2012), and Putman et al. (2012). Enzo uses an AMR grid to solve the equations of hydrodynamics, with this particular run using a version of Enzo before the uniform release of Enzo 2.0. Enzouses an FFT in the root grid gravity solver and a third-order piecewise parabolic method hydrodynamics solver. Feedback is thermal as described in Cen et al. (2005). The simulation includes metallicitydependent cooling to a temperature of $10 \mathrm{~K}$ (Dalgarno \& McCray 1972), neutral hydrogen shielding from UV radiation, and diffuse photoelectric heating (Abbott 1982; Joung et al. 2009).

\subsection{Art}

The Art (Kravtsov et al. 1997; Kravtsov 2003) run uses an AMR grid to solve the equations of hydrodynamics. Artuses a multilevel particle mesh gravity solver and a second-order Godunov method hydrodynamics solver. Our run uses the star formation and feedback models described in Ceverino et al. (2014) and includes thermal feedback from supernova explosions and stellar winds (Ceverino \& Klypin 2009; Ceverino et al. 2010) as well as radiative feedback (model RadPre_LS_IR in Ceverino et al. 2014). This model of radiative feedback includes radiation pressure from ionizing and infrared photons, photoheating, and photoionization from massive stars. Other recent papers using similar physics include Zolotov et al. (2015), Snyder et al. (2015a), Ceverino et al. (2015, 2016, 2017), Goerdt \& Ceverino (2015), Tacchella et al. (2016a, 2016b), and Tomassetti et al. (2016), Mandelker et al. (2017).

\subsection{Ramses}

The Ramses (Teyssier 2002) run (Ramses version 3.0) uses an AMR grid to solve the equations of hydrodynamics. Ramses uses a particle mesh gravity solver and a secondorder MUSCL scheme hydrodynamics solver. The gas cooling is based on a metallicity-dependent cooling, including metal line cooling down to a temperature floor of $100 \mathrm{~K}$. A stiffening of the interstellar medium (ISM) equation of state (chosen as a power law with $\gamma=4 / 3$ ) was used to prevent gas with densities higher than the 1 atom $/ \mathrm{cm}^{-3}$ threshold to cool further than $100 \mathrm{~K}$ and artificially fragment. Feedback includes energy from stellar winds and supernovae (deposited 
Table 1

Simulation Code Details

\begin{tabular}{|c|c|c|c|c|c|}
\hline & Enzo & Art & Ramses & Arepo & Gizmo-PSPH \\
\hline Gravity Solver & FFT in the root grid & Multilevel particle mesh & Multigrid particle mesh & $\begin{array}{l}\text { Tree multipole expansion parti- } \\
\text { cle mesh }\end{array}$ & $\begin{array}{l}\text { Tree multipole expansion parti- } \\
\text { cle mesh }\end{array}$ \\
\hline Hydrodynamics Solver & $\begin{array}{l}\text { Third-order piecewise parabolic } \\
\text { method }\end{array}$ & $\begin{array}{l}\text { Second-order Godunov } \\
\text { method }\end{array}$ & $\begin{array}{l}\text { Second-order MUSCL } \\
\text { scheme }\end{array}$ & Second-order MUSCL scheme ${ }^{a}$ & Pressure-energy SPH \\
\hline High Res. $m_{\mathrm{DM}}^{\mathrm{b}}$ & $1.75 \times 10^{5} M_{\odot}$ & $1.75 \times 10^{5} M_{\odot}$ & $1.75 \times 10^{5} M_{\odot}$ & $1.75 \times 10^{5} M_{\odot}$ & $1.75 \times 10^{5} M_{\odot}$ \\
\hline $\begin{array}{l}\text { Grav. Softening }\left[h^{-1} \text { comov- }\right. \\
\text { ing pc] }\end{array}$ & 95 (DM, gas) & 95 (DM, gas) & 95 (DM, gas) & 95 (DM, gas) & 95 (DM), 14 (gas) \\
\hline SF Threshold & $0.04 \mathrm{~cm}^{-3}$ & $1 \mathrm{~cm}^{-3}$ & $1 \mathrm{~cm}^{-3}$ & $0.13 \mathrm{~cm}^{-3}$ & $5 \mathrm{~cm}^{-3}+$ self-grav. + molecular \\
\hline SF Efficiency & $\epsilon=0.03$ & $\epsilon=0.03$ & $\epsilon=0.03$ & $t_{\mathrm{SFR}}=2.2 \mathrm{Gyr}$ & $\begin{array}{l}\epsilon=1 \text { (in self-grav., mole- } \\
\text { cular gas) }\end{array}$ \\
\hline Stellar Feedback ${ }^{\mathrm{c}}$ & Thermal & Thermal \& Rad. & Kinetic & Kinetic & Mixed [see text] \\
\hline Temperature Floor & $10 \mathrm{~K}$ & $300 \mathrm{~K}$ & $100 \mathrm{~K}$ & $500 \mathrm{~K}$ & $10 \mathrm{~K}$ \\
\hline UV Background & HM96 (increased Gaussian width) & HM96 & HM96 & FG09 & FG09 \\
\hline Reionization & $z=6$ & $z=7$ & $z=10$ & $z=10$ & $z=10$ \\
\hline
\end{tabular}

Notes. HM96-Haardt \& Madau (1996); FG09—Faucher-Giguère et al. (2009).

${ }^{a}$ Subsequent versions of Arepo have switched to a different time integration (Pakmor et al. 2016) using Heun's method.

${ }^{\mathrm{b}}$ For Lagrangian codes, high resolution gas particle mass is $3.3 \times 10^{4} M_{\odot}$.

${ }^{\mathrm{c}}$ See the text for detailed descriptions of feedback models. 
in kinetic form) following Dubois \& Teyssier (2008), where the proper distributions of SN II lifetimes are based on Leitherer et al. (1999, 2010), such that energy from SNe II is injected continuously between 2 and 50 Myr. Feedback from SNe Ia are also included, following Greggio \& Renzini (1983) to compute the SN frequency. This run has essentially the same physics as in Dubois et al. (2014), Welker et al. (2014), Codis et al. (2015), and Chisari et al. (2015), with the exception that we have not included any AGN physics here.

\subsection{Arepo}

The Arepo (Springel 2010) run employs a quasi-Lagrangian finite volume method for solving the hydrodynamic equations of motion (Vogelsberger et al. 2013). The version of Arepo used here employs a tree multipole expansion gravity solver and a second-order Godunov method hydrodynamics solver with a MUSCL scheme; however, subsequent versions of Arepo have switched to a different time integration in the hydrodynamics solver (Pakmor et al. 2016) using Heun's method. Radiative gas cooling includes both primordial cooling (Katz et al. 1996) as well as line cooling from heavy elements (Wiersma et al. 2009a; Vogelsberger et al. 2013). Pressurization of the ISM, star formation, and associated feedback is handled using the Springel \& Hernquist (2003) subgrid model. Time delayed stellar mass return and metal enrichment is carried out (Wiersma et al. 2009b; Vogelsberger et al. 2013), and kinetic star formation driven winds are employed with a wind velocity scaled to the local dark matter velocity dispersion. Winds are launched carrying $40 \%$ of the local ISM metallicity to prevent over-ejecting metal mass from the dense ISM (Zahid et al. 2014).

This run includes a physics implementation that is similar to that used in the Illustris simulation (Genel et al. 2014; Vogelsberger et al. 2014), with the notable difference that no AGN physics is included here. Other recent work that contains similar physics include Torrey et al. (2014, 2015a, 2015b), Wellons et al. (2015, 2016), Snyder et al. (2015b), RodriguezGomez et al. (2015), Sales et al. (2015), Bray et al. (2016), and Mistani et al. (2016).

\subsection{Gizmo-PSPH}

The Gizmo-PSPH (Hopkins 2015) run uses a tree multipole expansion for the gravity solver and the pressure-energy formulation of smoothed particle hydrodynamics (PSPH; Hopkins 2013) together with a number of additional improvements to artificial viscosity, timestepping, and higher-order kernels, to solve the equations of hydrodynamics. ${ }^{17}$ Radiative gas cooling includes both primordial cooling (Katz et al. 1996) as well as cooling from 11 separately tracked metal species (Wiersma et al. 2009a). Gas follows an ionized + atomic + molecular cooling curve from $T=10-10^{10} \mathrm{~K}$.

Star formation and feedback uses the Feedback In Realistic Environments (FIRE) prescriptions from Hopkins et al. (2014), which explicitly follow the mass, metal, momentum, and energy deposition by radiation pressure, photoionization and photoelectric heating, stellar winds, and SNe (Types II and Ia), with all rates tabulated from the stellar population model

\footnotetext{
17 Gizmo is a multi-methods code that gives the user the choice of several hydrodynamic methods. This is why we use the label Gizmo-PSPH throughout this work, to distinguish the PSPH implementation from alternate methods.
}

STARBURST99 (Leitherer et al. 1999) assuming a Kroupa (2001) IMF. They do not include AGN feedback. Unlike the other codes here, which assume stars form with a relatively low efficiency per free-fall time in all gas above some relatively large density threshold $\sim 0.1-1 \mathrm{~cm}^{-3}$, the FIRE models restrict star formation only to gas that is locally self-gravitating (following Hopkins et al. 2013), self-shielding and molecular (following Krumholz \& Gnedin 2011), Jeans-unstable, and exceeds a higher density $n>5 \mathrm{~cm}^{-3}$, but within this highly restricted gas assumes star formation occurs on a free-fall time. Other recent work that contains identical FIRE code and methods include Oñorbe et al. (2015), Chan et al. (2015), Ma et al. (2015), Faucher-Giguère et al. (2015), and Wheeler et al. (2015).

\section{Basic Halo Properties}

\subsection{Large-scale Structure and Mass Growth}

We begin with a visual inspection of the region around the galaxy for each simulation. Figure 1 shows the gas density (number density of $\mathrm{H}$; top) and density-weighted temperature (bottom) projections at $z=3$ through a cube of width 272 physical kpc ( $4 R_{\text {vir }}$ at this redshift). The top panels of Figure 1 show qualitative agreement between the simulations on the general geometry and structure of the forming disk galaxy at this redshift, as well as its placement in a large-scale cosmic filament that is continually delivering an inflow of cold gas into the virial radius of the galaxy. However, the detailed structure of the galaxy-and even that of the filament into which the galaxy is embedded-does appear to vary significantly between simulations. For example, the width of the cosmic filament, the size and structure of the galactic disk, and the peak density of infalling satellite galaxies all vary on a noticeable level.

Perhaps more striking is the temperature differences among simulations shown in the bottom panels of Figure 1. All simulations demonstrate the presence of a significant gaseous halo around the galaxy, as well as streams of filamentary gas that penetrate the halo and deposit cold gas in the inner halo, near the galactic region. However, the extent that feedback has enriched the CGM and IGM, the density structure of the gaseous halo, the temperature distribution of hot gas, and the precise structure of the cold flows as they interact with the gaseous halo of the galaxy vary significantly among simulations.

To illustrate some of the similarities and differences among the simulations, the two left panels of Figure 2 show the mass growth of the halo as a function of time, including the total mass (black) and galaxy stellar mass (yellow) on the leftmost panel, as well as the cold (blue) and hot (magenta) gas fractions within the virial radius. Note that the total virial mass (left panel) is quite similar among simulations, despite very different feedback implementations. Comparing the linear scale of the middle panel to the log scale of the left panel, we also note that the gas fractions (middle panel) are relatively similar among the simulations, although there are still noticeable variations. The average total/cold/hot gas fractions during the entire redshift range $z=3-1$ averaged over all the simulations is represented by the horizontal dotted lines in the figure. Not surprisingly (given the mass scale of this halo), all simulations show that the dominant supply of halo gas is in a cold phase, rather than a massive reservoir of hot gas. 


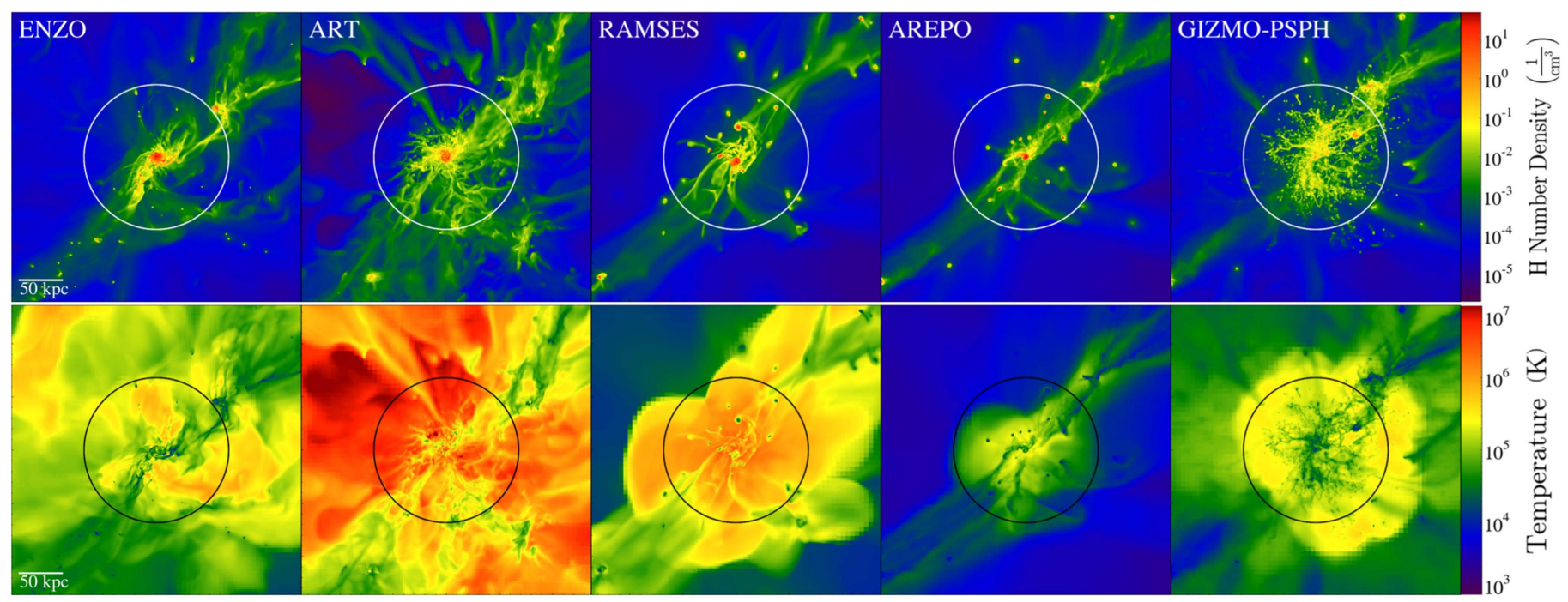

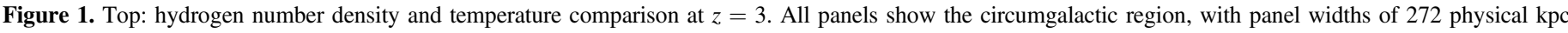

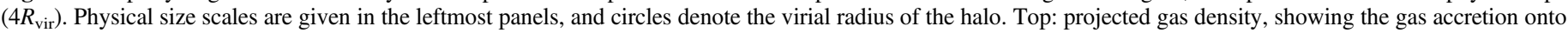

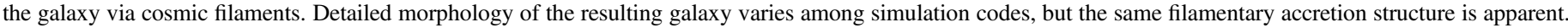

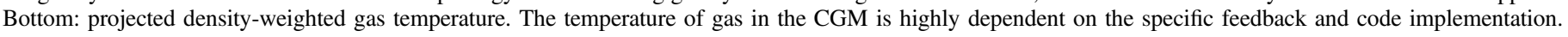

The total galaxy stellar mass (left panel) shows a much more significant variation among simulations, with the GizmoPSPH code in particular forming a much smaller stellar mass than any of the other codes used here, likely as a result of strong feedback implementations. As a comparison, the upper limit of baryonic mass (the virial mass times the cosmic baryon fraction) is shown here as a thin black dotted line, and the shaded cyan region shows the expected galaxy stellar mass range for the given virial mass based on abundance matching (median value $\pm 1 \sigma$ from Behroozi et al. 2013a). Interestingly, the galaxy stellar mass for Gizmo-PSPH is much closer to observational expectations, and may even be slightly underproducing stars in the simulated galaxy, rather than overproducing them, as in the other simulations. As our goal in this work is to focus on similarities between codes, with emphasis on the galaxy halos and not the galaxies themselves, we defer a more detailed discussion of the numerous differences between the simulations and their implications for galaxy formation as a topic for future study.

\subsection{Angular Momentum}

One fundamental result of the recent emerging picture of angular momentum acquisition in galaxies is that gas in the halos of galaxies tends to have specific angular momentum 3-5 times higher than the dark matter (Kimm et al. 2011; Stewart et al. 2011b, 2013; Danovich et al. 2015). We revisit these previous findings by comparing the spin parameter, $\lambda$, of both the cold halo gas and the dark matter in the halo for all our simulations. We adopt the spin parameter from Bullock et al. (2001): $\lambda_{x} \equiv j_{x} / \sqrt{2} V R$, where $\lambda_{x}$ is the spin parameter of a given component, based on that component's specific angular momentum, $j_{x}$, and $V$ and $R$ are defined by the virial velocity and virial radius of the halo, respectively. The right panel of Figure 2 shows the spin parameter for each saved output of each simulation between $z=3$ to $z=1$, where we only include material inside the virial radius but outside of the central region $\left(0.1<R / R_{\mathrm{vir}}<1.0\right)$ in our calculations since we are interested in the halo, not the galaxy itself. While the simulations vary in the precise value (and direction-not shown) of the angular momentum of their gaseous halos, we find several important qualitative agreements across all the simulations.

1. Cold halo gas (and hot halo gas-not shown in the figure, for clarity) consistently has more specific angular momentum than the dark matter component.

2. While simulations agree that dark matter halo spin parameters are typically $\lambda_{\mathrm{DM}} \sim 0.03$, the average cold halo gas spin parameter across our simulations is significantly higher: $\lambda_{\text {cold }} \simeq 0.12$.

3. In agreement with previous work, averaging over all simulations, the cold halo gas contains $\simeq 4$ times the specific angular momentum of the dark matter halo (though with considerable variation), while the hot gas typically has $\simeq 2$ times the specific angular momentum of the dark matter.

These findings confirm previous results: the angular momentum of galaxy halos varies significantly among components; the dark matter invariably measures a cumulative combination of past accretion, resulting in the lowest specific angular momentum; the hot gaseous halo is typically built and maintained both by non-filamentary "hot-mode" gas accretion, as well as feedback and outflows (which are sensitive to subgrid physics models); and the cold halo gas traces filamentary "cold-mode" accretion and has the highest specific angular momentum (Stewart et al. 2013). Thus, while our simulations agree with previous $N$-body simulations for a dark matter halo spin parameter, one should expect to observe typical cold halo gas with significantly higher angular momentum, with spin parameters $\lambda_{\text {cold }} \sim 0.1$.

In a previous study of four cosmological zoom-in simulations (all using the same hydrodynamic code), Stewart et al. (2013) found no significant trend between cold gas spin parameter and cosmological time (at least, not significant enough to be apparent with a non-statistical sample of high resolution zoom-in simulations). Therefore, while Figure 2 arguably shows a trend of increasing cold gas spin parameter from $z=3-1$, this may be a consequence of this particular 

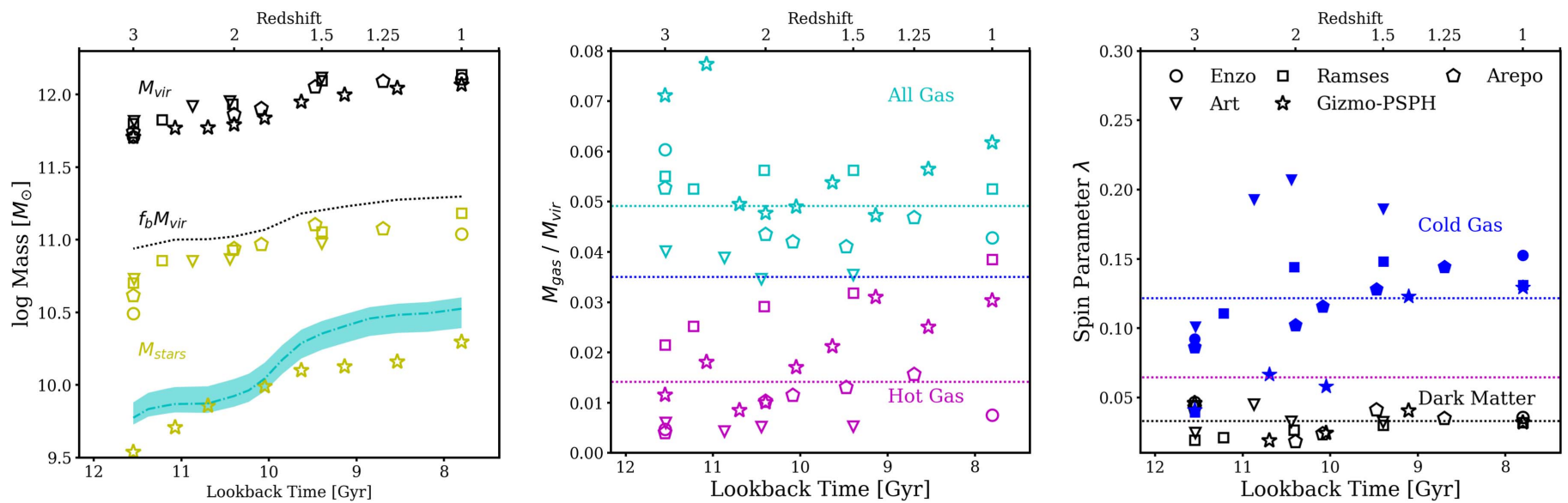

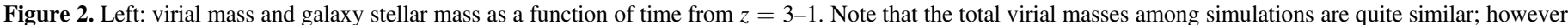

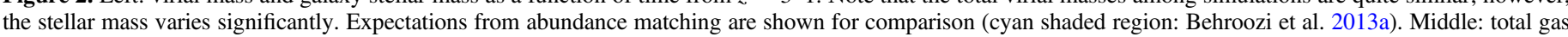

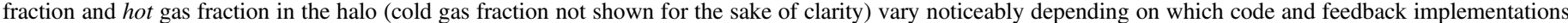

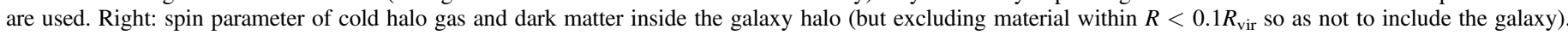

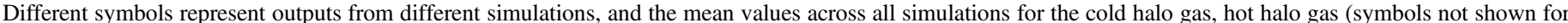

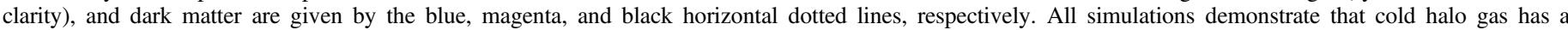

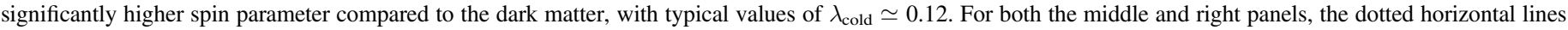
represent averages over the entire redshift range and across all simulations for all gas (cyan), cold gas (blue), hot gas (red), and/or dark matter (black).

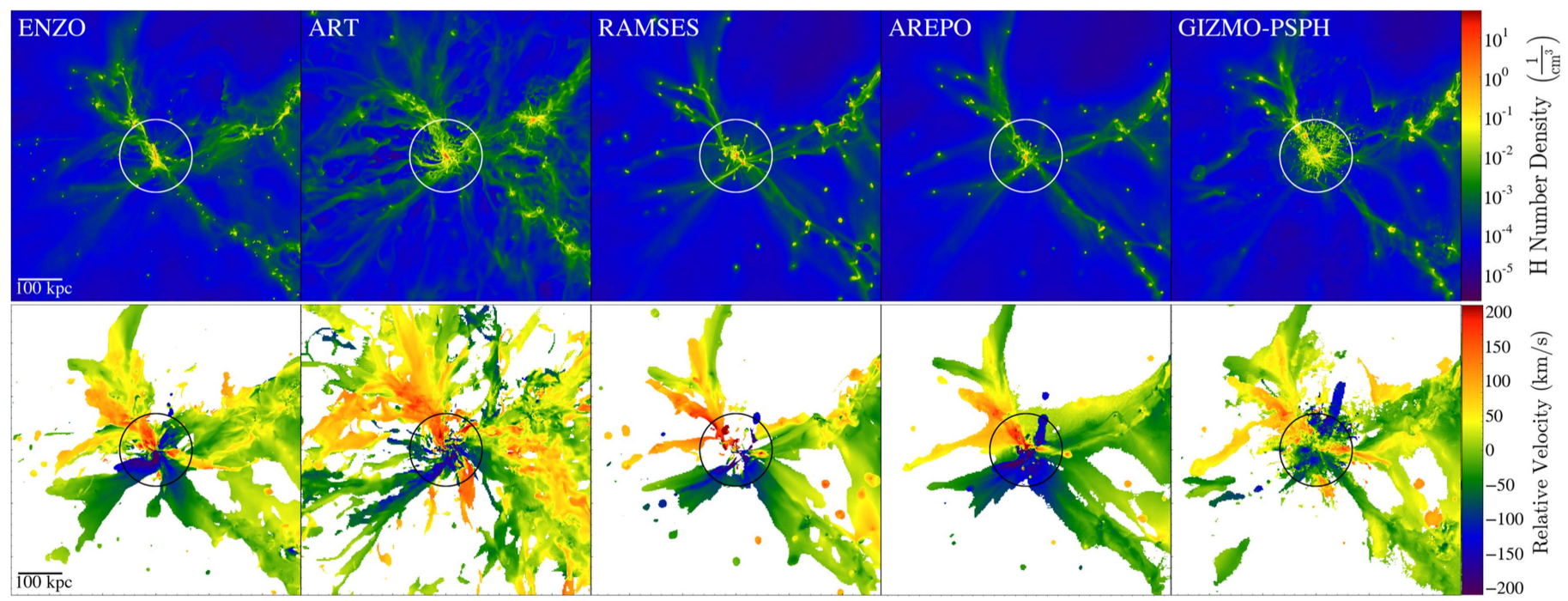

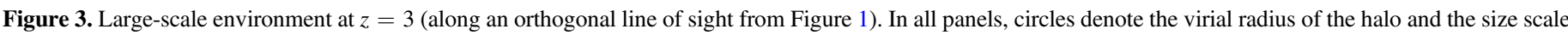

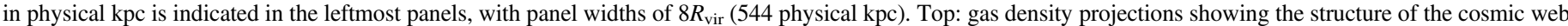

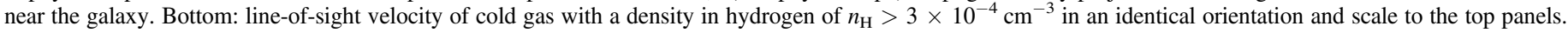

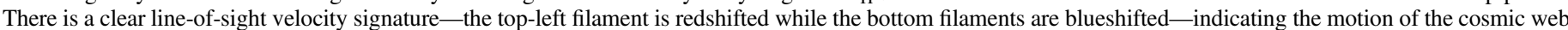

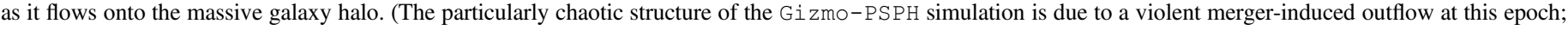
see Section 5.2.)

halo's unique merger and accretion history, and is not likely to be a general result of galaxy formation in LCDM.

\section{Large-scale Filamentary Inflow}

In order to place this high angular momentum cold halo gas in the proper cosmological context, Figure 3 shows the largescale environment around the simulated galaxy-where, for purposes of this work, we define the halo environment by box widths of $8 R_{\mathrm{vir}}(544$ physical $\mathrm{kpc}$ at $z=3$ ). The top panels again show the gas density ( $\mathrm{H}$ number density), similar to Figure 1 but zoomed out by a factor of two and viewed along an orthogonal orientation. The bottom panels show the line-ofsight velocity of all cold gas above a minimum density threshold in hydrogen (all forms) of $n_{\mathrm{H}}>3 \times 10^{-4} \mathrm{~cm}^{-3}$, which was chosen to select only gas sufficiently dense to be embedded in filamentary (or dark matter halo) structures in these large-scale environments.

Because the galaxy is the most massive halo in its environment (i.e., not a member of a group or cluster), the cosmic filaments in its environment are strongly affected by the halo potential, with gas, dark matter, and smaller galaxies all flowing along the filaments toward the galaxy, demonstrated by the clear line-of-sight velocity indications in the bottom panels. For example, the filament to the upper left of the galaxy (situated in front of the galaxy along this line of sight) consistently shows redshifted velocities in all simulations, while the two filaments below (and behind) the galaxy are consistently blueshifted. 


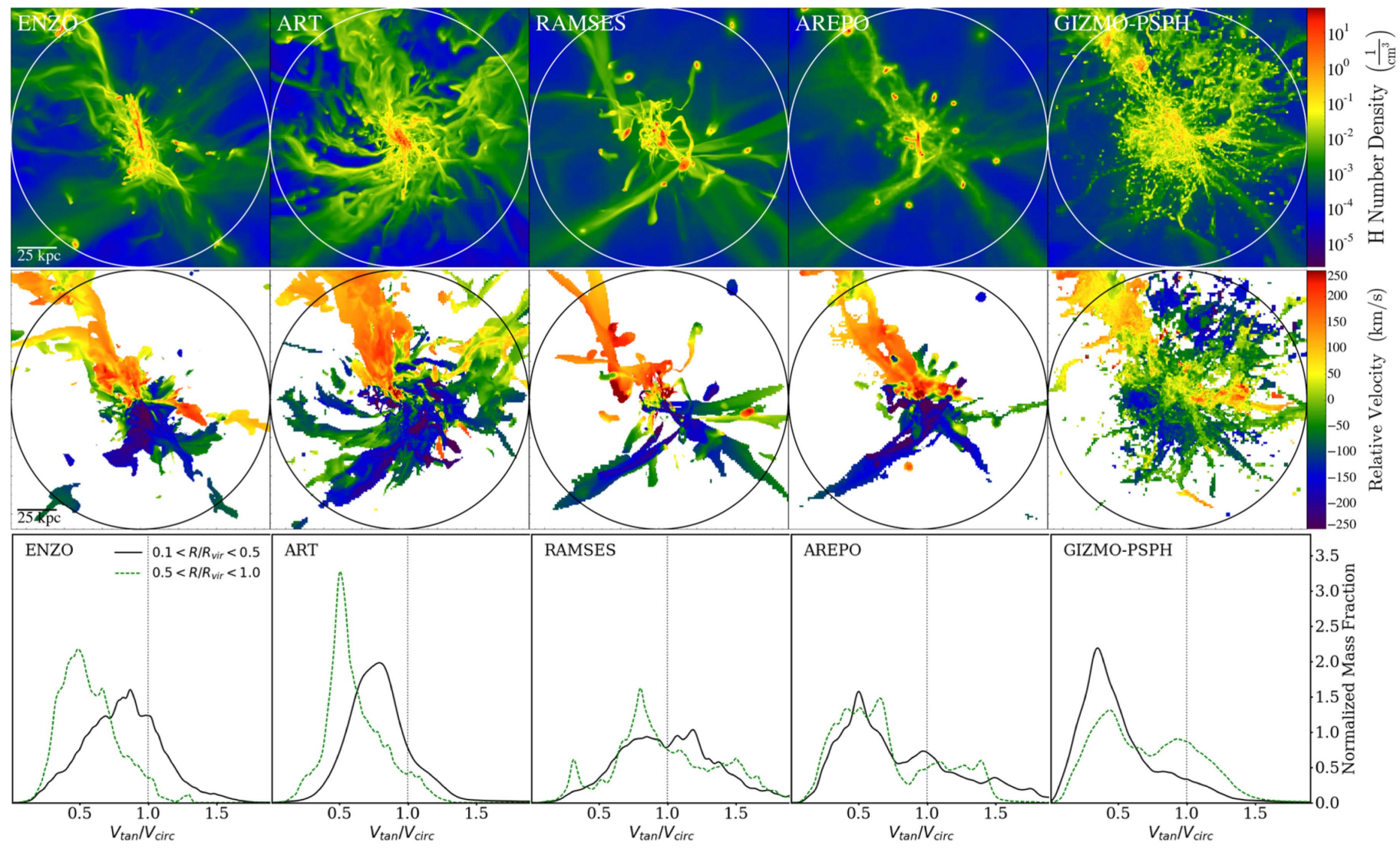

Figure 4. Top/middle: density projections and line-of-sight velocities at $z=3$, similar to Figure 3, except that panels have now been "zoomed in" to the virial radius of the halo, and the minimum density threshold for hydrogen gas in the bottom panels has been increased by a factor of 10 from Figure 3 to $n_{\mathrm{H}}>3 \times 10^{-3} \mathrm{~cm}^{-3}$ (corresponding to column densities of $N_{\mathrm{H}} \gtrsim 10^{17} \mathrm{~cm}^{-2}$ ). Circles denote the virial radius of the halo and the size scale in physical kpc is indicated in the leftmost panels. The kinematics of the co-directional inspiraling cold streams appear linked to the large-scale filaments that are fueling them, as seen in Figure 3 . (See Section 5.2 regarding the chaotic structure of the Gizmo-PSPH simulation.) Bottom: $V_{\mathrm{tan}} / V_{\text {circ }}$ at radius $R / R_{v i r}$ as a function of $R / R_{\mathrm{vir}}$ for cold dense gas in the halo. Most cold dense gas in the halo spirals in toward the center of the halo, and is not angular-momentum supported.

This result is perhaps not surprising, as any three-dimensional filamentary structure where matter flows along the cosmic web toward a central overdensity (and is viewed along an arbitrary axis) is unlikely to show multiple filaments all flowing perpendicular to the line of sight. Thus, one should naively expect strong line-of-sight velocities to be apparent when viewing large-scale filamentary gas flows. Although this may not be a surprising result, it is important to keep these large-scale gas flows in mind for future discussion of the kinematics of inspiraling cold streams in the galaxy's halo. We will see in Section 5 that these large-scale filamentary flows have a direct impact on the behavior of the cold gas within the virial radius of the halo.

Note that the line-of-sight velocity structure of the filament flowing in from the right of the galaxy shows considerably more variation between the simulations. This occurs because this filament does happen to be roughly perpendicular to the line of sight. Thus, the velocities along the rightmost filament are more sensitive to the peculiar velocities of galaxies, gas streams, and outflows, which vary more strongly between simulations than the gross large-scale flows toward the central halo.

\section{Inspiraling Cold Streams}

We begin investigating the morphology and kinematics of cold halo gas in Figure 4, which is analogous to Figure 3, except that the panels now focus only on material within the virial radius (panel widths of 136 physical $\mathrm{kpc}$ at $z=3$ ). The bottom panels again show line-of-sight velocity maps of cold dense gas $^{18}$ in the halo, except that we have increased the minimum density threshold by a factor of 10 when compared to Figure 3, to a hydrogen density of $n_{\mathrm{H}}>3 \times 10^{-3} \mathrm{~cm}^{-3}$ (this should correspond to a minimum hydrogen column density of $N_{\mathrm{H} \mathrm{I}} \gtrsim 10^{17} \mathrm{~cm}^{-2}$; Schaye 2001; Altay et al. 2011).

The exact morphology of gas in the halo of the galaxy varies considerably among the simulations, which is not surprising, given the vastly different feedback mechanisms implemented in each simulation, some of which drive explosive spherical outflows that violently shred the ISM and CGM of the galaxy (e.g., Gizmo-PSPH) and some of which instead drive highvelocity bi-conical outflows out of the plane of the galaxy (e.g., Enzo). However, we also note that some of the morphological differences may also be influenced by the precise timing of galaxy mergers. For example, the Gizmo-PSPH simulation is in the midst of a violent outflow at this epoch, due to a recent merger, which partially explains the significantly more chaotic structure shown in Figure 4 (we will demonstrate in Section 5.2

\footnotetext{
${ }^{18}$ We select gas based on temperature and density rather than $\mathrm{H}$ I content or species column density because we want to avoid any differences in ionization fractions among simulations when making our comparison. The qualitative trend that there is always high-angular momentum inspiraling gas in the halo does not depend on the details of this selection criterion, though quantitative measures (e.g., the apparent covering fraction of this gas) will of course depend on these details-a topic we plan to revisit in future work.
} 
that the Gizmo-PSPH simulation's line-of-sight velocity structure is much more similar to the other simulations immediately before and after this merger-driven outflow event). While the same general merger and accretion history takes place for each simulation, the exact timing of these mergers at a given epoch may vary, and any coherent velocity structure for cold gas in the galaxy's halo is typically destroyed during a sufficiently strong outflow event.

Despite these varied differences in morphology, the middle panels of Figure 4 show a similar qualitative picture. As was the case with the large-scale environment, the cold gas entering the virial radius from the upper-left filament shows a dramatic redshift in each simulation, while the cold gas entering from the bottom filaments show strong blueshifts. (As before, the lineof-sight velocity of the material in the upper-right quadrant of these panels is less uniform, as it probes a gas accreting along a filament that is roughly perpendicular to the line of sight.) The bottom panels of Figure 4 show that while the angular momentum content of the halo gas is high, the bulk of this inflowing gas does not have enough angular momentum to be fully rotationally supported. That is, most - but certainly not all - of the cold dense halo gas at $R>0.1 R_{\text {vir }}$ has a tangential velocity $V_{\tan } / V_{\text {circ }}<1$ (where $V_{\text {circ }}=\sqrt{G M(<R) / R}$ is the circular velocity at a given radius). Thus, despite the clear velocity structure shown in the middle panels of the figure, this high angular momentum gas should not be considered rotationally supported, but rather spiraling in toward the center of the halo, consistent with the short "sinking times" of $\sim 1-2$ halo dynamical times previously reported by Stewart et al. (2011b).

The qualitative result in each case is a clear co-directional inflow signature, with cold dense halo gas easily divided by a single cutting plane into the redshifted versus blueshifted half of the halo, flowing through the halo via a chaotic assortment of high angular momentum inspiraling cold streams that are kinematically linked to inflow from the cosmic web.

Similar structures have been noted a number of times in the literature, but with a variety of terminologies, including the "messy region" (Ceverino et al. 2010), "cold flow disks" (Stewart et al. 2011b, 2013), the "AM sphere" (Danovich et al. 2012), or "extended rings" (Danovich et al. 2015). Indeed, depending on the simulation code utilized, one can easily see in Figure 4 how the kinematics and morphology of the inspiraling streams may or may not be well-described as a "messy region" (e.g., Ramses) or a more orderly disk-like structure (e.g., Art). Thus, while we find that the exact morphologyincluding size, orientation, clumpiness, thickness-of any structure that results from the inspiraling cold streams may vary significantly among simulations, each code does produces a qualitatively similar picture in which there is a clear line-ofsight velocity structure within the virial radius of the halo that is kinematically linked to that of the large-scale filamentary environment of Figure 3 (with the exception of the GizmoPSPH simulation at this epoch; see Section 5.2).

Figure 5 shows line-of-sight velocity maps (along an orthogonal orientation) for dense cold gas at $z=2$ (top), $z=1.5$ (middle), and $z=1$ (bottom) for various subsets of the simulation runs (as labeled). The left panels of this figure look at the large-scale environment (analogous to Figure 3. While the basic filamentary nature of the gaseous inflows becomes less apparent at decreasing redshift (when the filaments are less dense), we can still note the same qualitative behavior of inflowing gas. On environmental scales, filamentary inflow results in the same clear line-of-sight velocity signature as before; across all simulations, gas flowing into the virial radius from the top of the panels is blueshifted, while gas flowing in from the bottom is redshifted, with the only notable exception being the Ramses code at $z=1$, which is likely the result of the lack of self-shielding from the UV background, leaving very little cold gas above our minimum density threshold, so almost no cold dense inflow is still visible in the figure. While the detailed structure of the inflowing gas again varies among simulations, it seems apparent that filamentary gas accretion along a three-dimensional cosmic web onto an overdense region (at this mass scale in the redshift range $1<z<3$ ) tends to produce the same qualitative picture across all the simulations, regardless of the subgrid physics.

The right panels of Figure 5 shows an analogous line-ofsight velocity analysis, but zoomed-in to the halo virial radius for $z=2$ (top), $z=1.5$ (middle), and $z=1$ (bottom), and again increasing the density threshold by a factor of 10 (similar to Figure 4). Again, the precise structure of the inspiraling cold streams varies among the simulations, but most of the simulations produce qualitatively similar pictures; there continues to be a clear large-scale velocity structure within the virial radius of the halo that is kinematically linked to the largescale filamentary inflow shown in the left panels, again with the exception of Ramses at $z=1$, which has evacuated most of its halo of cold dense gas altogether.

\subsection{Co-directional Halo Gas}

As a means of quantifying this result, we define the "codirectional mass fraction" in the following way, at any given epoch. For three arbitrary orthogonal projections, we define a cutting plane (passing through the center of the halo) that best divides the halo into positive versus negative line-of-sight velocities for all gas within the virial radius. Each particle (or cell, depending on code architecture) can then be defined as co-directional (along this projection) if its line-of-sight velocity was correctly categorized by this cutting plane. ${ }^{19} \mathrm{We}$ then select the projection with the highest overall co-directional fraction for all gas in the halo (but not the galaxy: $0.1<$ $R / R_{\mathrm{vir}}<1.0$ ). This selection typically corresponds to the projection in which the galaxy is seen closest to edge-on, though we note that this may not always be the case, if there is a significant misalignment between the angular momentum direction of the inspiraling gas and that of the galactic disk. The co-directional mass fraction of any given component (dark matter, cold gas, hot gas, or all gas) is the mass fraction that has been categorized as co-directional along this preferred projection.

Using this definition, the left panel of Figure 6 shows the codirectional mass fraction as a function of radius at $z=3$ (for all gas versus dark matter). As might be expected, the average codirectional mass fraction for dark matter (taking into account all the simulations) is $\sim 50 \%$, though with considerable variation depending on the exact orientation of the co-directional cutting plane. However, among all five simulations, the co-directional mass fraction for gas shows remarkably similar behavior with radius_-declining smoothly from $\sim 85 \%$ at the galactic region

\footnotetext{
${ }^{19}$ If the halo gas were rotationally supported, we would say the gas is corotating rather than co-directional; however, we are hesitant to use this terminology, since the gas is actively spiraling inwards to the center of the halo, and co-rotation might be misinterpreted to imply angular momentum support.
} 

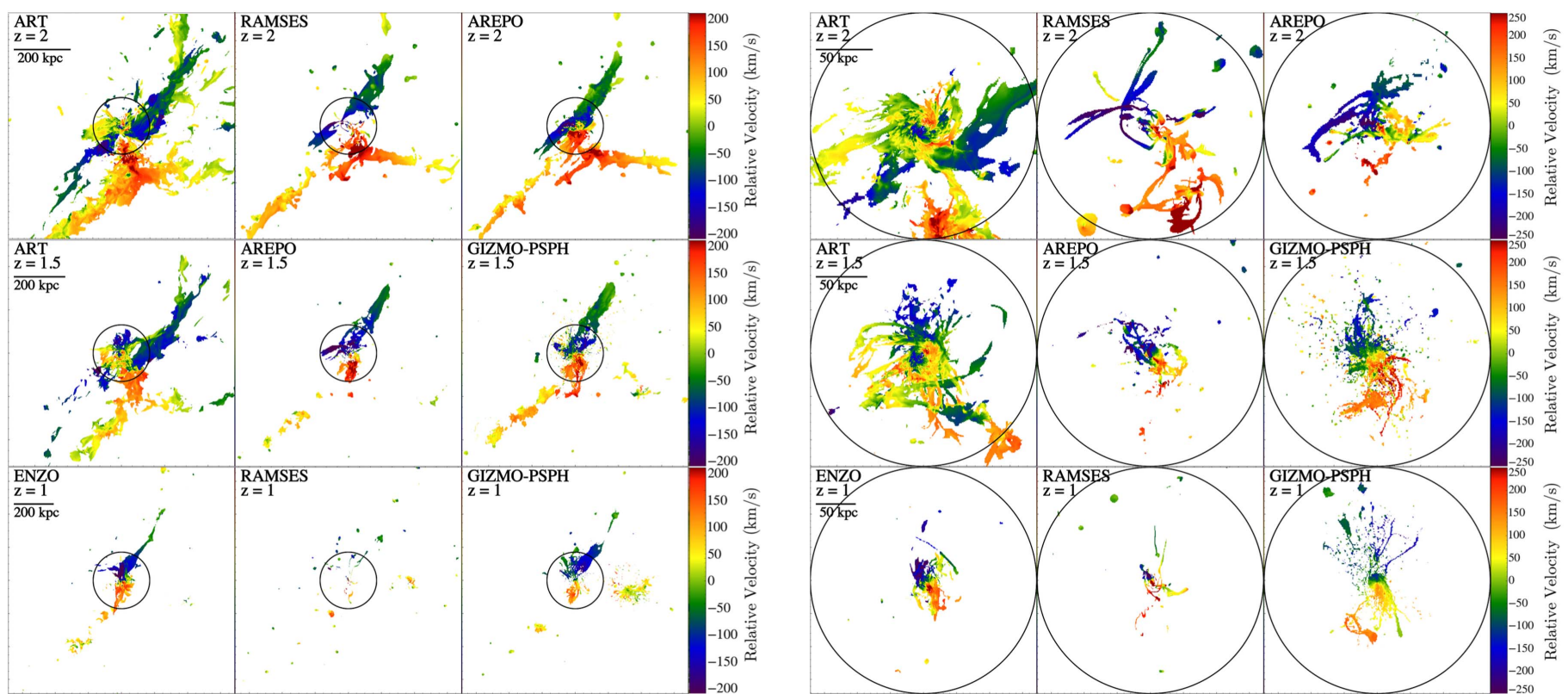

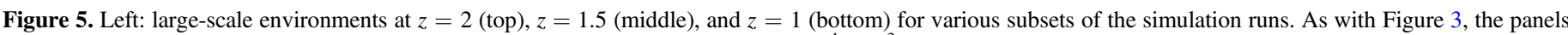

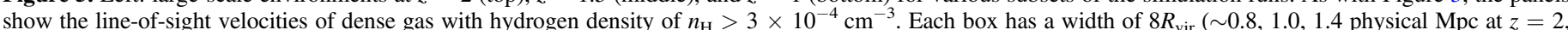

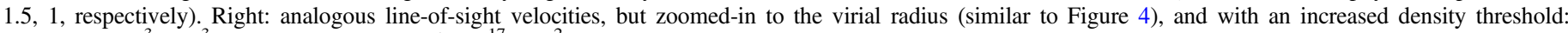

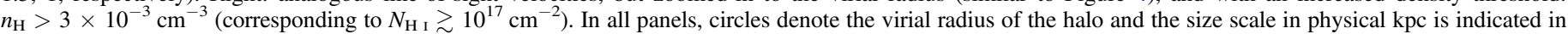

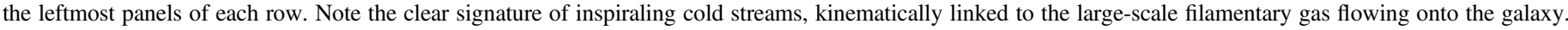

( $R=0.1 R_{\text {vir }}$ ) to $\sim 70 \%$ at the virial radius. Indeed, even extending to $\sim 2.5 R_{\mathrm{vir}}$, the co-directional mass fraction of gas in the cosmic web remains significantly higher than that of the dark matter, as expected if the line-of-sight velocity structure of the cold gas is kinematically linked to the filamentary gas flows beyond the virial radius of the halo.

We explore the co-directional mass fraction over cosmic time in the middle and right panels of Figure 6, which use the same procedure outlined above to compute a single value at each epoch for the total co-directional mass fraction in the halo (but not the galaxy: $0.1<R / R_{\mathrm{vir}}<1.0$ ) for dark matter versus gas (middle panel), and further distinguishing between cold gas versus hot gas (right panel). In both panels, the average for dark matter, all gas, cold gas, and hot gas among all simulations and over the entire redshift range from $z=3-1$ are given by the horizontal dotted lines. As shown in the figure, the dark matter co-directional mass fraction varies somewhat sporadically around $\sim 50 \%$, depending on the cutting plane orientation, while the halo gas shows significantly higher co-directional mass fractions (75\% for all halo gas). While there are significant variations in the detailed results among the simulations, with some codes showing stronger co-directional kinematics than others, all simulations also demonstrate a higher tendency for cold gas to show this co-directional velocity structure in the halo over hot gas (79\% versus $64 \%)$.

Since the halo gas mass is dominated by its cold component (see Figure 2), it is worth exploring whether the above trend in co-directional mass fraction between the cold and hot components might be the result of an offset in angular momentum direction between the hot versus cold gaseous halos. After repeating the above analysis, but using the line-ofsight velocity of the hot gas to define the co-directional cutting plane, we find only small variations in the above results. For example, the co-directional mass fraction in the halo at $z=3$ for [Enzo, Art, Ramses, Arepo, Gizmo-PSPH] decreases slightly (if at all) for cold gas from [84\%, 82\%, 81\%, 72\%,
$69 \%$ ] to $[79 \%, 81 \%, 81 \%, 72 \%, 69 \%]$, respectively, and increases slightly (if at all) for hot gas from [53\%, 62\%, 65\%, $58 \%, 64 \%]$ to $[55 \%, 62 \%, 68 \%, 58 \%, 64 \%]$. Therefore, we conclude that the overall trends shown in Figure 6 are not highly sensitive to the way we define the co-directional cutting plane.

Taken together with our previous, more qualitative results, our findings suggest that across a broad range in hydrodynamic code types and subgrid physics models of galaxy formation, the presence of co-directional inspiraling cold streams in galaxy halos at $z>1$ is a natural consequence of high angular momentum filamentary inflow along the cosmic web, and represents a robust prediction of cosmological gas accretion in LCDM.

\subsection{The Rapid Destruction and Re-formation of Coherent Inspiraling Gas at $\mathrm{z}=3$}

In the discussion of Figures 3 and 4, we noted that the velocity structure of the galaxy in the Gizmo-PSPH simulation is not nearly as clean and orderly as the other simulations at $z=3$, and therefore does not seem to host the same clear codirectional velocity structure. While we thought it important to show all galaxies at precisely the same epoch, we note that in Gizmo-PSPH, the galaxy happens to be in the midst of a postmerger starburst, accompanied by a violent outflow event at this epoch, due to this code's strong feedback physics. Halohalo mergers, of course, tend to occur at broadly similar times in all codes, but differences in galaxy masses and halo baryonic mass distributions mean that the galaxy-galaxy mergers can and do occur at significantly different times, and with different mass ratios and corresponding consequences for star formation, at the halo center (see, e.g., Stewart et al. 2009; Hopkins et al. 2010). The obvious clumpiness of the outflows may owe, at least partially, to well-known numerical difficulties capturing fluid-mixing instabilities in SPH, even in the improved P-SPH 

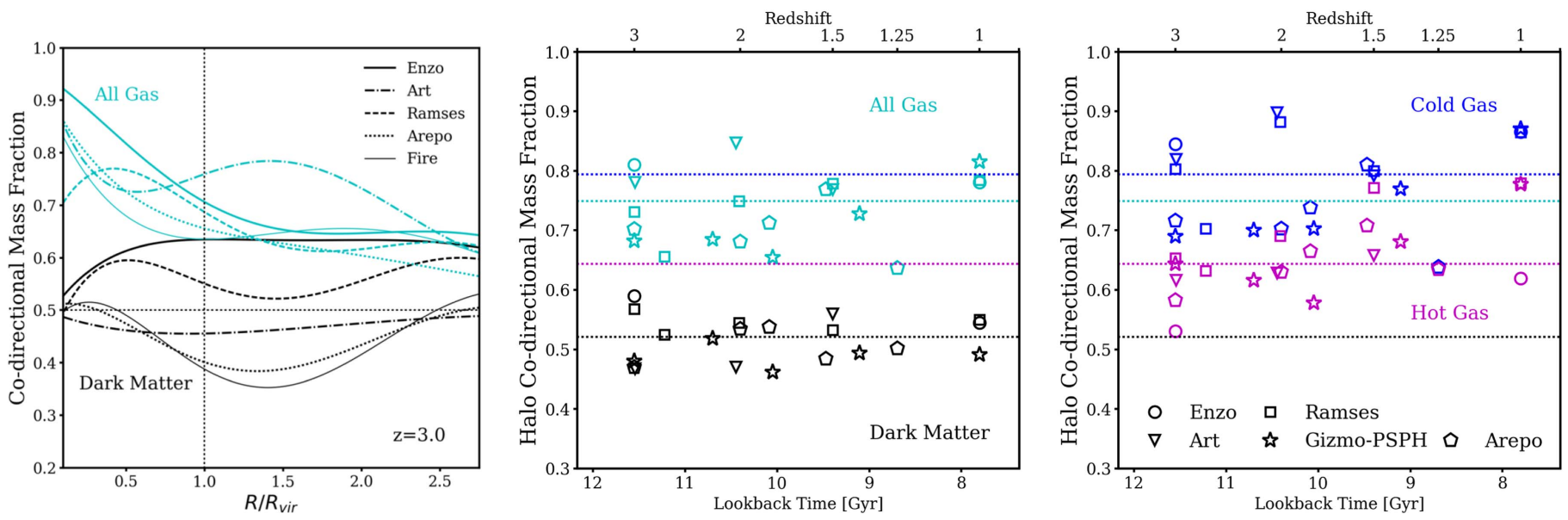

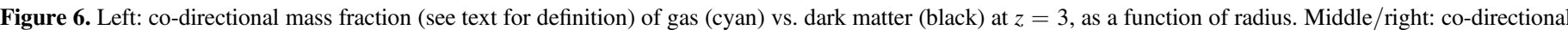

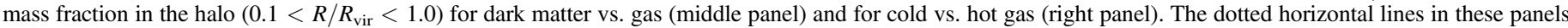
represent averages over the entire redshift range and across all simulations for all gas (cyan), cold gas (blue), hot gas (red), and dark matter (black).

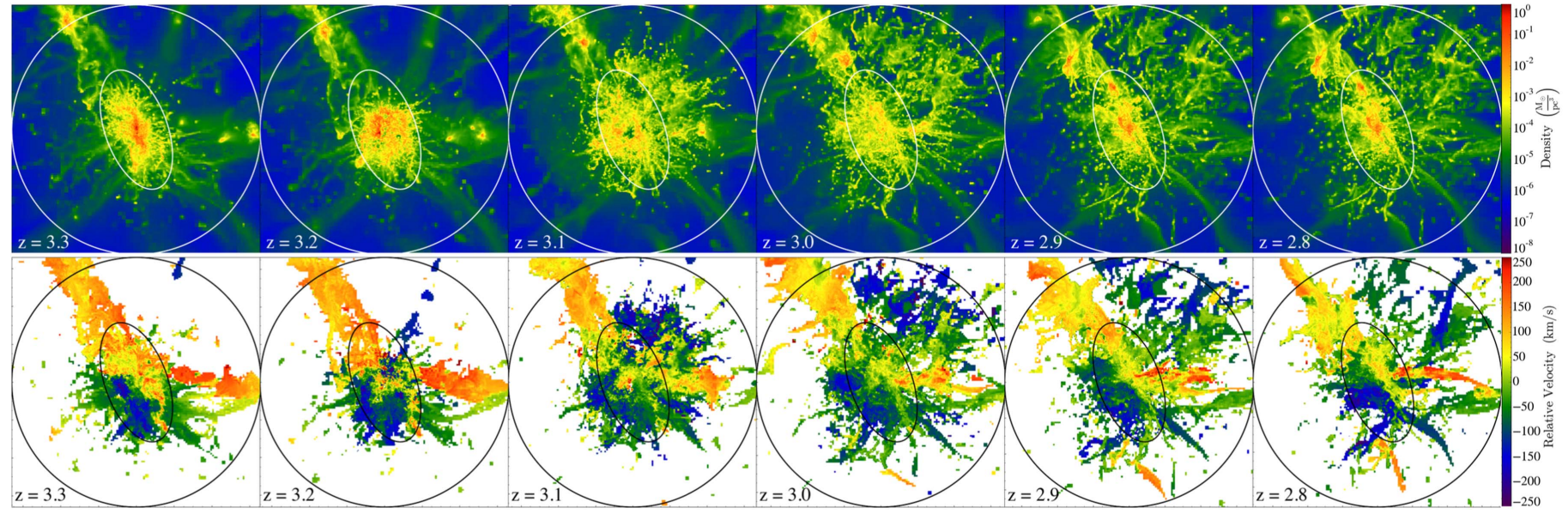

Figure 7. Time lapse of a post-merger violent outflow event in Gizmo-PSPH at $z \sim 3$, proceeding from left to right. The overlaid circles denote the halo virial radius and the overlaid ellipse in each panel roughly corresponds to the region of coherent inspiraling gas at $z=3.3$, to aid the eye in comparison between images. Top: density map of the gas in the halo. Bottom: line-of-sight velocity of cold dense gas in the halo (identical analysis to Figure 4). The coherent rotation in the bottom-left panel is effectively destroyed by the violent outflow from $z=3.2-3.0$, but once the outflow event is over, fresh high angular momentum infall along the cosmic web begins to establish a new coherent inspiraling region by $z=2.8$, demonstrating the robustness of the inspiraling gas phenomenon.

implementation; this is supported by early results from the FIRE-2 simulations, which use a different, mesh-free Godunov-type finite volume method to solve the hydrodynamics (P. Hopkins 2017, private communication).

In Figure 7, we show the structure of this galaxy immediately before and after this violent merger event. The time sequence begins in the left panel at $z=3.3$, where inflowing cold gas demonstrates the same line-of-sight velocity structure as in Figure 4, including the presence of co-directional inspiraling cold streams, which have initially taken the apparent form of an extended disk-like structure reminiscent of the "cold flow disks" reported previously in cosmological simulations (e.g., Stewart et al. 2011b). To aid the eye in comparing the images, some of which are quite chaotic during the outflow, an identical ellipse has been overlaid on each image roughly corresponding to this coherent inspiraling gas region. At this epoch, the recent influx of fresh gas onto the central regions of the galaxy results in a spike in star formation, and consequently a violent spherical outflow event from $z=3.2-3.0$ that effectively destroys the ISM of the galaxy (leaving a deficit of gas in the center of the galaxy, as seen at $z=3.1$ ) and disrupting the inflowing filamentary gas in the CGM of the halo. However, the filamentary gas continues to flow into the halo, and this inflow continues to contribute substantial angular momentum. As a result, a new co-directional velocity structure becomes apparent almost immediately after the outflow event has subsided, with the co-directional mass fraction of cold dense gas in the halo (defined as described in Section 5.1) changing rapidly from $81 \%$ at $z=3.3$ (before the outflow) to $\sim 60 \%$ during the outflow, back up to $76 \%$ at $z=2.8$ (postmerger). By the rightmost panels, the co-directional inspiraling cold streams have once again formed a roughly disk-like structure, along a very similar orientation to the original inspiraling gas structure. We argue that the bursty nature (Muratov et al. 2015) of the subgrid physics as implemented in Gizmo-PSPH coupled with this demonstration of the nearimmediate regrowth of the coherent inspiraling gas structure after a massive outflow event only reinforces the robust nature of inspiraling cold streams in the halos of massive galaxies in LCDM. 


\section{Discussion}

Past studies of galaxy formation simulations have reported the existence of co-rotating structures of cool gas in the outskirts of galaxy halos. Our results indicate that inspiraling halo gas of this kind is robust to different feedback models and hydrodynamic solvers. One implication of this result is that extended, high-angular momentum cold stream configurations offer a testable observational signature of the LCDM galaxy formation paradigm. For example, the fact that a large fraction of the halo gas have velocities that are co-directional means that observations of the gas (e.g., from quasar absorption systems) will show a blueshifted and redshifted side that is usually interpreted as rotation. We emphasize that in this case most of the gas is far from being angular-momentum supported and that "inspiraling" halo gas is a more accurate description of its coherent motion than "rotating."

Encouragingly, there is a growing body of observational evidence that seems to indicate that co-directional halo gas is indeed seen around real galaxies. For example, kinematic studies of some Ly $\alpha$ nebulae suggest rotational velocities and inflow rates consistent with those expected for these inspiraling streams (Martin et al. 2014; Prescott et al. 2015). Similarly, absorption line studies are beginning to emphasize the bimodal distribution of absorption detections, where detections along the galaxy's minor axis tend to show absorption properties consistent with outflowing gas, while detections roughly along the galaxy's major axis demonstrate properties (such as corotational inflow) that are consistent with inspiraling cold streams (Kacprzak et al. 2010, 2012a, 2012b; Bouché et al. 2012, 2013; Crighton et al. 2013; Nielsen et al. 2015; Bouché et al. 2016; Ho et al. 2017).

Perhaps the most direct confirmation of the existence of inspiraling halo gas comes from Martin et al. (2015), who performed a spectroscopic analysis on the cosmic filament (illuminated by two nearby QSOs) first detected by Cantalupo et al. (2014) at $z \sim 2$. They found that a substantial fraction of the illuminated region was in fact a huge co-rotating gaseous structure. The extremely extended gaseous "disk" (extending to $\sim R_{\text {vir }} / 2$, corresponding to a width of 125 physical kpc) showed smooth rotation kinematics, with one side of the disk kinematically linked to the inflow velocity of the nearby cosmic filament. This very closely resembles what we have presented here for co-directional inspiraling cold streams, though we note that the particular system observed by Martin et al. (2015) was estimated to be a much more massive halo than what we have simulated here $\left(M_{\text {vir }} \sim 10^{13} M_{\odot}\right)$, and it therefore reported a correspondingly more massive and extended protogalactic disk than found in our simulations, as might be expected for a larger, more massive halo. A similar cold flow protodisk, again fed by a cosmic filament that was first detected in Ly $\alpha$ emission, was also reported in Martin et al. (2016), suggesting that inspiraling disk-like structures may be common phenomena for massive galaxies at high redshift.

While not seen in our particular simulations, we also speculate that polar ring galaxies-which have previously been suggested as evidence of cold flow gas accretion onto galaxies (Macciò et al. 2006; Brook et al. 2008; Spavone et al. 2010)_may be a result of a similar phenomenon. Such galaxies could reasonably occur when strong central torques (e.g., from a major galaxy merger) result in a near perpendicular misalignment between the angular momentum of the central galaxy and that of the inflowing cold-mode gas.

We note that the inspiraling cold streams in our simulations are significantly more massive and extended (relative to the halo virial radius) at high redshift, when cosmic filaments are more narrowly defined and contain higher density gas flows. However, Figure 2 and previous work (e.g., Stewart et al. 2013) both demonstrate that accreting cold gas continues to have high angular momentum, even at later times where the rotational signature of a continuous gaseous structure may be less clear. Additionally, Figure 6 demonstrates that the codirectional mass fraction of cold gas in the halo stayed consistently high over cosmic time (at least until $z=1$ ). We speculate that it may be possible that this high angular momentum accretion helps to explain observations of extended XUV disks (e.g., Thilker et al. 2005, 2007; Lemonias et al. 2011; Holwerda et al. 2012), local extended H I disks (e.g., García-Ruiz et al. 2002; Oosterloo et al. 2007; Christlein \& Zaritsky 2008; Sancisi et al. 2008; Walter et al. 2008; Wang et al. 2013; Huang et al. 2014; Courtois et al. 2015), and corotating cold halo gas around local Milky Way analogs (e.g., Diamond-Stanic et al. 2016).

Indeed, these growing observations of high angular momentum material in the outskirts of galaxy halos would be quite difficult to explain if one were to assume the canonical picture of galaxy formation whereby baryons in galaxy halos share the same distribution of angular momentum as the dark matter. In contrast, the cold flow paradigm naturally predicts that halo gas (and particularly the cold halo gas) preferentially constitutes recent gas accretion from the cosmic web, with $\sim 3-5$ times the angular momentum of the dark matter, naturally explaining the kinds of high angular momentum phenomena being observed. We caution, however, that we have not focused on the gaseous halos in our simulations at $z<1$ here, and leave a more detailed comparison between simulations and low-z observations as a topic of further study.

\section{Conclusion}

We have simulated the evolution of a Milky Way-sized galaxy from identical cosmological initial conditions with a variety of simulation codes: Enzo, Art, Ramses, Arepo, and Gizmo-PSPH. Each code has used subgrid physics models drawn from scientific literature common to each simulation type, and we have compared the simulations in an attempt to draw robust conclusions about galaxy formation in LCDM (focusing on $z>1$ ) that are not sensitive to uncertain aspects of galaxy formation simulations. To ensure uniform analysis among the various code types, we have used the hydrodynamic analysis software yt, which enables the same analysis routine to be performed on each code.

While we found many aspects of the simulated galaxies that did vary substantially among the simulations (e.g., morphology, stellar mass, hot gas halo temperature, and mass, to be discussed further in future work), we found the following qualitative features common to all simulations, regardless of which subgrid physics model or hydrodynamic code was used:

1. Gas in the galaxy halo has substantially higher specific angular momentum than the dark matter in the halo, with mean values of $j_{\text {cold }} \simeq 4 j_{\text {DM }}$ and $j_{\text {hot }} \simeq 2 j_{\text {DM }}$ (though with considerable scatter), leading to a typical cold halo gas spin parameter of $\lambda_{\text {cold }} \simeq 0.12$. 
2. The large-scale filamentary structure is qualitatively similar in all simulations (with minor variations, for example regarding lower mass streams of secondary importance to the galaxy's growth). The three-dimensional geometry of these filaments, which are all flowing toward the central galaxy (the highest overdensity in its environment) results in a strong line-of-sight velocity structure. Filaments flowing onto the galaxy from opposite directions (along an arbitrary line of sight) tend to show alternating blueshifted and redshifted velocities relative to the galaxy as they flow toward the galaxy center.

3. As the filamentary gas accretion enters the virial radius, the large-scale velocity structure of the accreting filaments inevitably results in inspiraling cold streams in the halo of the galaxy that carry significant angular momentum as they spiral in from the virial radius to the galactic region. For the Milky Way-sized halo simulated here, the maximum line-of-sight velocity expected for these inspiraling streams is $\sim 250 \mathrm{~km} \mathrm{~s}^{-1}$ (corresponding to roughly 1.5 times the virial velocity of the halo). As a result, the vast majority ( $\sim 80 \%$ ) of cold halo gas follows a clear co-directional velocity structure (with a single cutting plane dividing positive versus negative line-ofsight velocities) as the cold streams spiral toward the center of the halo. In contrast, the co-directional mass fractions in the halo are considerably lower for the hot gas $(\sim 65 \%)$ or the dark matter $(\sim 50 \%)$.

4. Inspiraling cold streams occasionally take the previously reported morphology of cold flow disks: high angular momentum cold gas that is transitioning from the cosmic web, though the halo as a roughly disk-like structure (except that the gas is not angular-momentum supported) and will eventually accrete onto the galactic disk. These coherent inspiraling structures represent continuous and dynamic flows from the cosmic web; even after a violent outflow event disrupts the CGM in one of the simulations, the newly inflowing gas rapidly re-forms a similar inspiraling structure along the same orientation soon after the outflow has subsided.

In this work, we have limited our analysis to the growth of a single Milky Way-sized halo at $z>1$ using a variety of different hydrodynamic codes and feedback physics implementations. It is therefore difficult to draw general conclusions about galaxy formation from the simulation of a single halo; however, a number of theoretical works have previously established the high angular momentum nature of filamentary gas accretion, using various hydrodynamic codes, larger cosmological volumes, and/or analysis of multiple zoom-in simulations. For example, Pichon et al. (2011) analyzed $\sim 15,000$ halos at $z>1.5$ from a (lower resolution) cosmological-scale simulation using the Ramses code; in a companion work, Kimm et al. (2011) also included $~ 900$ intermediateresolution halos and two high-resolution zoom-in simulations to $z=0$ using Ramses; Stewart et al. (2011b, 2013) analyzed four zoom-in simulations to $z=0$ using the SPH code Gasoline; and Danovich et al. (2015) analyzed 29 zoom-in simulations at $z>1.5$ using the Art code. The results presented here demonstrate that the high angular momentum nature of cold gas accretion in LCDM is not likely to change (in the qualitative sense) among a broad range of different physics implementations and hydrodynamic codes, suggesting that the presence of inspiraling cold streams in galaxy-size halos appears to be a robust expectation of LCDM.

However, we note that there are considerable variations in the quantitative nature (morphology, rotational velocity, size, temperature, density, etc.) of the inspiraling cold streams in each of the simulations performed in this work. The detailed properties and prevalence of these inspiraling streams are yet to be fully understood, and cannot be determined from the single high-resolution simulation presented here. The co-directional velocity structure noted here is also likely to depend on the geometry and kinematics of the cosmic web in the galaxy's environment, so we speculate that there are likely to be significant environment effects, even at fixed halo mass. For example, Milky Way-sized galaxies near the outskirts of galaxy clusters would not be expected to dominate the gravitational potential of the cosmic web in their large-scale environment, so we may not expect to find the same clear co-directional velocity signature for filamentary inflow (i.e., Figure 3) for such systems either.

We also take special note that in several of the simulation codes used here (and in previous works), these inspiraling streams result in the formation of transient inspiraling disk-like structures qualitatively similar to the "cold flow disks" of, e.g., Stewart et al. (2013). The exploration of the prevalence of these inspiraling gaseous structures in simulations, for different environments and halo masses, would be a useful topic of further study, especially in light of recent observations of large co-rotating gaseous structures at $z \sim 2$ (Martin et al. 2015, 2016) that are strikingly similar to the qualitative results presented here.

Computations described in this work were performed using the publicly available Enzo code (http://enzo-project.org), and the publicly available yt toolkit (http://yt-project.org/), both of which are the products of collaborative efforts of many independent scientists from numerous institutions around the world. Their commitment to open science has helped make this work possible. K.R.S. would especially like to thank Matt Turk and Nathan Goldbaum for their support with yt. K.R.S. also thanks Volker Springel, Lars Hernquist, and Paul Torrey for running the Arepo simulation discussed in this work, for allowing us to include its results as part of this comparison project, and for providing useful discussions and comments.

K.R.S. was supported by HST-GO-14268.026-A. J.S.B. was supported by HST AR-12836. C.A.F.G. was supported by NSF grants AST-1412836 and AST-1517491, by NASA grant NNX15AB22G, and by STScI grants HST-AR-14293.001-A and HST-GO-14268.022-A. J.D. acknowledges support from the Spin(e) grant ANR-13-BS05-0005 of the French Agence Nationale de la Recherche (http://cosmicorigin.org). Support for P.F.H. was provided by an Alfred P. Sloan Research Fellowship, NASA ATP Grant NNX14AH35G, and NSF Collaborative Research Grant \#1411920 and CAREER grant \#1455342. The Art run was performed at the National Energy Research Scientific Computing Center (NERSC) at Lawrence Berkeley National Laboratory. DC acknowledges support from the European Research Council under the European Community's Seventh Framework Programme (FP7/2007-2013) via the ERC Advanced Grant "STARLIGHT: Formation of the First Stars" (project number 339177). The Ramses simulation 
was performed on the DiRAC Facility, jointly funded by BIS and STFC. J.D.'s research is partly funded by Adrian Beecroft and the Oxford Martin School. The Gizmo-PSPH run was carried out on the CUNY HPC which is supported, in part, under National Science Foundation Grants CNS-0958379, CNS-0855217, and ACI-1126113, and the City University of New York High Performance Computing Center at the College of Staten Island. Storage and analysis of the simulations was performed on the CTP cluster, which is supported by GRTI grant CT04AGR15001 and the Physics Division of the U.S. Army Research Office grant \#64775-PH-REP.

\section{References}

Abbott, D. C. 1982, ApJ, 263, 723

Agertz, O., \& Kravtsov, A. V. 2016, ApJ, 824, 79

Agertz, O., Kravtsov, A. V., Leitner, S. N., \& Gnedin, N. Y. 2013, ApJ, 770, 25

Agertz, O., Moore, B., Stadel, J., et al. 2007, MNRAS, 380, 963

Agertz, O., Teyssier, R., \& Moore, B. 2009, MNRAS, 397, L64

Altay, G., Theuns, T., Schaye, J., Crighton, N. H. M., \& Dalla Vecchia, C. 2011, ApJL, 737, L37

Avila-Reese, V., Colín, P., Gottlöber, S., Firmani, C., \& Maulbetsch, C. 2005, ApJ, 634, 51

Behroozi, P. S., Wechsler, R. H., \& Conroy, C. 2013a, ApJ, 770, 57

Behroozi, P. S., Wechsler, R. H., \& Wu, H.-Y. 2013b, ApJ, 762, 109

Bertschinger, E. 2011, GRAFIC-2: Multiscale Gaussian Random Fields for Cosmological Simulations, Astrophysics Source Code Library

Bett, P., Eke, V., Frenk, C. S., Jenkins, A., \& Okamoto, T. 2010, MNRAS, 404, 1137

Binney, J. 1977, ApJ, 215, 483

Bouché, N., Finley, H., Schroetter, I., et al. 2016, ApJ, 820, 121

Bouché, N., Hohensee, W., Vargas, R., et al. 2012, MNRAS, 426, 801

Bouché, N., Murphy, M. T., Kacprzak, G. G., et al. 2013, Sci, 341, 50

Bray, A. D., Pillepich, A., Sales, L. V., et al. 2016, MNRAS, 455, 185

Brook, C. B., Governato, F., Quinn, T., et al. 2008, ApJ, 689, 678

Brook, C. B., Governato, F., Roškar, R., et al. 2011, MNRAS, 415, 1051

Brooks, A. M., Governato, F., Quinn, T., Brook, C. B., \& Wadsley, J. 2009, ApJ, 694, 396

Bryan, G. L., Norman, M. L., O'Shea, B. W., et al. 2014, ApJS, 211, 19

Bullock, J. S., Dekel, A., Kolatt, T. S., et al. 2001, ApJ, 555, 240

Cantalupo, S., Arrigoni-Battaia, F., Prochaska, J. X., Hennawi, J. F., \& Madau, P. 2014, Natur, 506, 63

Cen, R., Nagamine, K., \& Ostriker, J. P. 2005, ApJ, 635, 86

Ceverino, D., Arribas, S., Colina, L., et al. 2016, MNRAS, 460, 2731

Ceverino, D., Dekel, A., \& Bournaud, F. 2010, MNRAS, 404, 2151

Ceverino, D., \& Klypin, A. 2009, ApJ, 695, 292

Ceverino, D., Klypin, A., Klimek, E. S., et al. 2014, MNRAS, 442, 1545

Ceverino, D., Primack, J., \& Dekel, A. 2015, MNRAS, 453, 408

Ceverino, D., Primack, J., Dekel, A., \& Kassin, S. A. 2017, MNRAS, 467, 2664

Chan, T. K., Keres, D., Oñorbe, J., et al. 2015, MNRAS, 454, 2981

Chen, D. N., Jing, Y. P., \& Yoshikaw, K. 2003, ApJ, 597, 35

Chisari, N., Codis, S., Laigle, C., et al. 2015, MNRAS, 454, 2736

Christlein, D., \& Zaritsky, D. 2008, ApJ, 680, 1053

Codis, S., Pichon, C., Devriendt, J., et al. 2012, MNRAS, 427, 3320

Codis, S., Pichon, C., \& Pogosyan, D. 2015, MNRAS, 452, 3369

Courtois, H. M., Zaritsky, D., Sorce, J. G., \& Pomarède, D. 2015, MNRAS, 448, 1767

Crighton, N. H. M., Hennawi, J. F., \& Prochaska, J. X. 2013, ApJL, 776, L18

Cullen, L., \& Dehnen, W. 2010, MNRAS, 408, 669

Dalgarno, A., \& McCray, R. A. 1972, ARA\&A, 10, 375

Danovich, M., Dekel, A., Hahn, O., Ceverino, D., \& Primack, J. 2015, MNRAS, 449, 2087

Danovich, M., Dekel, A., Hahn, O., \& Teyssier, R. 2012, MNRAS, 422, 1732 Dekel, A., \& Birnboim, Y. 2006, MNRAS, 368, 2

Dekel, A., Birnboim, Y., Engel, G., et al. 2009, Natur, 457, 451

Diamond-Stanic, A. M., Coil, A. L., Moustakas, J., et al. 2016, ApJ, 824, 24

D’Onghia, E., \& Navarro, J. F. 2007, MNRAS, 380, L58

Dubois, Y., Pichon, C., Welker, C., et al. 2014, MNRAS, 444, 1453

Dubois, Y., \& Teyssier, R. 2008, A\&A, 477, 79

Fall, S. M., \& Efstathiou, G. 1980, MNRAS, 193, 189
Faucher-Giguère, C.-A., Hopkins, P. F., Kereš, D., et al. 2015, MNRAS, 449, 987

Faucher-Giguère, C.-A., \& Kereš, D. 2011, MNRAS, 412, L118

Faucher-Giguère, C.-A., Kereš, D., \& Ma, C.-P. 2011, MNRAS, 417, 2982

Faucher-Giguère, C.-A., Lidz, A., Zaldarriaga, M., \& Hernquist, L. 2009, ApJ, 703, 1416

Fernández, X., Joung, M. R., \& Putman, M. E. 2012, ApJ, 749, 181

García-Ruiz, I., Sancisi, R., \& Kuijken, K. 2002, A\&A, 394, 769

Genel, S., Fall, S. M., Hernquist, L., et al. 2015, ApJL, 804, L40

Genel, S., Vogelsberger, M., Springel, V., et al. 2014, MNRAS, 445, 175

Gnedin, O. Y., Ceverino, D., Gnedin, N. Y., et al. 2011, arXiv:1108.5736

Goerdt, T., \& Ceverino, D. 2015, MNRAS, 450, 3359

Greggio, L., \& Renzini, A. 1983, A\&A, 118, 217

Haardt, F., \& Madau, P. 1996, ApJ, 461, 20

Hahn, O., Teyssier, R., \& Carollo, C. M. 2010, MNRAS, 405, 274

Ho, S. H., Martin, C. L., Kacprzak, G. G., \& Churchill, C. W. 2017, ApJ, 835,267

Hobbs, A., Read, J., \& Nicola, A. 2015, MNRAS, 452, 3593

Holwerda, B. W., Pirzkal, N., \& Heiner, J. S. 2012, MNRAS, 427, 3159

Hopkins, P. F. 2013, MNRAS, 428, 2840

Hopkins, P. F. 2015, MNRAS, 450, 53

Hopkins, P. F., Croton, D., Bundy, K., et al. 2010, ApJ, 724, 915

Hopkins, P. F., Keres, D., Oñorbe, J., et al. 2014, MNRAS, 445, 581

Hopkins, P. F., Narayanan, D., \& Murray, N. 2013, MNRAS, 432, 2647

Huang, S., Haynes, M. P., Giovanelli, R., et al. 2014, ApJ, 793, 40

Ishiyama, T., Rieder, S., Makino, J., et al. 2013, ApJ, 767, 146

Joung, M. R., Mac Low, M.-M., \& Bryan, G. L. 2009, ApJ, 704, 137

Joung, M. R., Putman, M. E., Bryan, G. L., Fernández, X., \& Peek, J. E. G. 2012, ApJ, 759, 137

Kacprzak, G. G., Churchill, C. W., Ceverino, D., et al. 2010, ApJ, 711, 533

Kacprzak, G. G., Churchill, C. W., \& Nielsen, N. M. 2012a, ApJL, 760, L7

Kacprzak, G. G., Churchill, C. W., Steidel, C. C., Spitler, L. R., \& Holtzman, J. A. 2012b, MNRAS, 427, 3029

Katz, N., Weinberg, D. H., \& Hernquist, L. 1996, ApJS, 105, 19

Kay, S. T., Pearce, F. R., Frenk, C. S., \& Jenkins, A. 2002, MNRAS, 330, 113

Kereš, D., \& Hernquist, L. 2009, ApJL, 700, L1

Kereš, D., Katz, N., Fardal, M., Davé, R., \& Weinberg, D. H. 2009, MNRAS, 395,160

Kereš, D., Katz, N., Weinberg, D. H., \& Davé, R. 2005, MNRAS, 363, 2

Kim, J., Choi, Y.-Y., Kim, S. S., \& Lee, J.-E. 2015, ApJS, 220, 4

Kim, J.-h., Abel, T., Agertz, O., et al. 2014, ApJS, 210, 14

Kimm, T., Devriendt, J., Slyz, A., et al. 2011, arXiv:1106.0538

Komatsu, E., Dunkley, J., Nolta, M. R., et al. 2009, ApJS, 180, 330

Kravtsov, A. V. 2003, ApJL, 590, L1

Kravtsov, A. V., Klypin, A. A., \& Khokhlov, A. M. 1997, ApJS, 111, 73

Kroupa, P. 2001, MNRAS, 322, 231

Krumholz, M. R., \& Gnedin, N. Y. 2011, ApJ, 729, 36

Leitherer, C., Ortiz Otalvaro, P. A., Bresolin, F., et al. 2010, ApJS, 189, 309

Leitherer, C., Schaerer, D., Goldader, J. D., et al. 1999, ApJS, 123, 3

Lemonias, J. J., Schiminovich, D., Thilker, D., et al. 2011, ApJ, 733, 74

Ma, X., Kasen, D., Hopkins, P. F., et al. 2015, MNRAS, 453, 960

Macciò, A. V., Moore, B., \& Stadel, J. 2006, ApJL, 636, L25

Maller, A. H., \& Bullock, J. S. 2004, MNRAS, 355, 694

Maller, A. H., \& Dekel, A. 2002, MNRAS, 335, 487

Maller, A. H., Dekel, A., \& Somerville, R. 2002, MNRAS, 329, 423

Mandelker, N., Dekel, A., Ceverino, D., et al. 2017, MNRAS, 464, 635

Marasco, A., Debattista, V. P., Fraternali, F., et al. 2015, MNRAS, 451, 4223

Martin, D. C., Chang, D., Matuszewski, M., et al. 2014, ApJ, 786, 107

Martin, D. C., Matuszewski, M., Morrissey, P., et al. 2015, Natur, 524, 192

Martin, D. C., Matuszewski, M., Morrissey, P., et al. 2016, ApJL, 824, L5

Martizzi, D., Teyssier, R., Moore, B., \& Wentz, T. 2012, MNRAS, 422, 3081

Mistani, P. A., Sales, L. V., Pillepich, A., et al. 2016, MNRAS, 455, 2323

Mo, H. J., Mao, S., \& White, S. D. M. 1998, MNRAS, 295, 319

Morris, J. P. 1996, PASA, 13, 97

Muñoz-Cuartas, J. C., Macciò, A. V., Gottlöber, S., \& Dutton, A. A. 2011, MNRAS, 411,584

Muratov, A. L., Keres, D., Faucher-Giguere, C.-A., et al. 2015, MNRAS, 454, 2691

Nelson, D., Genel, S., Pillepich, A., et al. 2016, MNRAS, 460, 2881

Nelson, D., Genel, S., Vogelsberger, M., et al. 2015, MNRAS, 448, 59

Nelson, D., Vogelsberger, M., Genel, S., et al. 2013, MNRAS, 429, 3353

Nielsen, N. M., Churchill, C. W., Kacprzak, G. G., Murphy, M. T., \& Evans, J. L. 2015, ApJ, 812, 83

Ocvirk, P., Pichon, C., \& Teyssier, R. 2008, MNRAS, 390, 1326

Oñorbe, J., Boylan-Kolchin, M., Bullock, J. S., et al. 2015, MNRAS, 454, 2092 
Oosterloo, T. A., Morganti, R., Sadler, E. M., van der Hulst, T., \& Serra, P. 2007, A\&A, 465, 787

Pakmor, R., Springel, V., Bauer, A., et al. 2016, MNRAS, 455, 1134

Pichon, C., Pogosyan, D., Kimm, T., et al. 2011, MNRAS, 418, 2493

Piontek, F., \& Steinmetz, M. 2011, MNRAS, 410, 2625

Prescott, M. K. M., Martin, C. L., \& Dey, A. 2015, ApJ, 799, 62

Prieto, J., Jimenez, R., Haiman, Z., \& González, R. E. 2015, MNRAS, 452 784

Putman, M. E., Peek, J. E. G., \& Joung, M. R. 2012, ARA\&A, 50, 491

Rees, M. J., \& Ostriker, J. P. 1977, MNRAS, 179, 541

Richardson, M. L. A., Scannapieco, E., Devriendt, J., et al. 2016, ApJ, 825, 83

Rodriguez-Gomez, V., Genel, S., Vogelsberger, M., et al. 2015, MNRAS, 449, 49

Sales, L. V., Vogelsberger, M., Genel, S., et al. 2015, MNRAS, 447, L6

Sancisi, R., Fraternali, F., Oosterloo, T., \& van der Hulst, T. 2008, A\&ARv, 15,189

Scannapieco, C., Wadepuhl, M., Parry, O. H., et al. 2012, MNRAS, 423, 1726

Schaye, J. 2001, ApJL, 562, L95

Sharma, S., \& Steinmetz, M. 2005, ApJ, 628, 21

Silk, J. 1977, ApJ, 211, 638

Snyder, G. F., Lotz, J., Moody, C., et al. 2015a, MNRAS, 451, 4290

Snyder, G. F., Torrey, P., Lotz, J. M., et al. 2015b, MNRAS, 454, 1886

Spavone, M., Iodice, E., Arnaboldi, M., et al. 2010, ApJ, 714, 1081

Springel, V. 2010, MNRAS, 401, 791

Springel, V., \& Hernquist, L. 2003, MNRAS, 339, 289

Stewart, K. R. 2016, arXiv:1612.00513

Stewart, K. R., Bullock, J. S., Wechsler, R. H., \& Maller, A. H. 2009, ApJ, 702, 307

Stewart, K. R., Brooks, A. M., Bullock, J. S., et al. 2013, ApJ, 769, 74

Stewart, K. R., Kaufmann, T., Bullock, J. S., et al. 2011a, ApJ, 738, 39

Stewart, K. R., Kaufmann, T., Bullock, J. S., et al. 2011b, ApJL, 735, L1

Tacchella, S., Dekel, A., Carollo, C. M., et al. 2016a, MNRAS, 458, 242

Tacchella, S., Dekel, A., Carollo, C. M., et al. 2016b, MNRAS, 457, 2790

Teyssier, R. 2002, A\&A, 385, 337

Thacker, R. J., \& Couchman, H. M. P. 2000, ApJ, 545, 728

Thilker, D. A., Bianchi, L., Boissier, S., et al. 2005, ApJL, 619, L79
Thilker, D. A., Bianchi, L., Meurer, G., et al. 2007, ApJS, 173, 538

Tillson, H., Devriendt, J., Slyz, A., Miller, L., \& Pichon, C. 2015, MNRAS, 449, 4363

Tomassetti, M., Dekel, A., Mandelker, N., et al. 2016, MNRAS, 458, 4477

Torrey, P., Snyder, G. F., Vogelsberger, M., et al. 2015a, MNRAS, 447, 2753

Torrey, P., Vogelsberger, M., Genel, S., et al. 2014, MNRAS, 438, 1985

Torrey, P., Vogelsberger, M., Sijacki, D., Springel, V., \& Hernquist, L. 2012, MNRAS, 427, 2224

Torrey, P., Wellons, S., Machado, F., et al. 2015b, MNRAS, 454, 2770

Trowland, H. E., Lewis, G. F., \& Bland-Hawthorn, J. 2013, ApJ, 762, 72

Turk, M. J. 2013, arXiv:1301.7064

Turk, M. J., \& Smith, B. D. 2011, arXiv:1112.4482

Turk, M. J., Smith, B. D., Oishi, J. S., et al. 2011, ApJS, 192, 9

van de Voort, F., Davis, T. A., Keres, D., et al. 2015, MNRAS, 451, 3269

van de Voort, F., Schaye, J., Booth, C. M., Haas, M. R., \& Dalla Vecchia, C. 2011, MNRAS, 414, 2458

Vitvitska, M., Klypin, A. A., Kravtsov, A. V., et al. 2002, ApJ, 581, 799

Vogelsberger, M., Genel, S., Sijacki, D., et al. 2013, MNRAS, 436, 3031

Vogelsberger, M., Genel, S., Springel, V., et al. 2014, MNRAS, 444, 1518

Wadsley, J. W., Veeravalli, G., \& Couchman, H. M. P. 2008, MNRAS, 387,427

Walter, F., Brinks, E., de Blok, W. J. G., et al. 2008, AJ, 136, 2563

Wang, J., Kauffmann, G., Jozsa, G. I. G., et al. 2013, MNRAS, 433, 270

Welker, C., Devriendt, J., Dubois, Y., Pichon, C., \& Peirani, S. 2014, MNRAS, 445, L46

Wellons, S., Torrey, P., Ma, C.-P., et al. 2015, MNRAS, 449, 361

Wellons, S., Torrey, P., Ma, C.-P., et al. 2016, MNRAS, 456, 1030

Wheeler, C., Oñorbe, J., Bullock, J. S., et al. 2015, MNRAS, 453, 1305

White, S. D. M., \& Frenk, C. S. 1991, ApJ, 379, 52

White, S. D. M., \& Rees, M. J. 1978, MNRAS, 183, 341

Wiersma, R. P. C., Schaye, J., \& Smith, B. D. 2009a, MNRAS, 393, 99

Wiersma, R. P. C., Schaye, J., Theuns, T., Dalla Vecchia, C., \& Tornatore, L. 2009b, MNRAS, 399, 574

Zahid, H. J., Torrey, P., Vogelsberger, M., et al. 2014, Ap\&SS, 349, 873

Zolotov, A., Dekel, A., Mandelker, N., et al. 2015, MNRAS, 450, 2327 